\title{
Velocity-resolved Reverberation Mapping of Five Bright Seyfert 1 Galaxies
}

\author{
G. De Rosa ${ }^{1,2,3}$ (1) M. M. Fausnaugh ${ }^{1}$, C. J. Grier ${ }^{1,4,5}$ (1) B. M. Peterson ${ }^{1,2,3}$ (1) K. D. Denney ${ }^{1,2,6,58}$, Keith Horne ${ }^{7}$ (1),
} M. C. Bentz ${ }^{8}$ (10) S. Ciroi ${ }^{9}$, E. Dalla Bontà,10, M. D. Joner ${ }^{11}$, S. Kaspi ${ }^{12,13}$, C. S. Kochanek ${ }^{1,2}$ (1) R. W. Pogge ${ }^{1,2}$ (1), S. G. Sergeev ${ }^{14}$, M. Vestergaard ${ }^{15,16}$ (10 , S. M. Adams ${ }^{1,17}$, J. Antognini ${ }^{1,18}$, C. Araya Salvo ${ }^{1}$, E. Armstrong ${ }^{19,20}$, J. Bae ${ }^{21,22,59}$ (i), A. J. Barth ${ }^{23}$ (1), T. G. Beatty ${ }^{1,4,24}$ (D) A. Bhattacharjee ${ }^{25,26}$, G. A. Borman ${ }^{14}$, T. A. Boroson ${ }^{27}$ (1) , M. C. Bottorff ${ }^{28}$, J. E. Brown ${ }^{29}$, J. S. Brown ${ }^{1}$ (1),

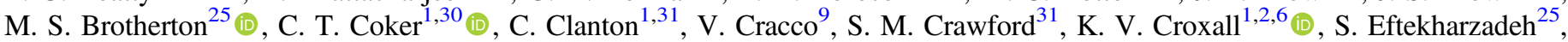
M. Eracleous ${ }^{4,5}$ (1) , S. L. Fiorenza ${ }^{32}$, A. Frassati ${ }^{9}$, K. Hawkins ${ }^{19,32}$ (1) , C. B. Henderson ${ }^{1,30}$ (1) , T. W.-S. Holoien ${ }^{1,2}$ (1)

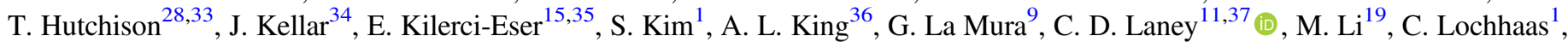
Z. $\mathrm{Ma}^{29}$, F. MacInnis ${ }^{28}$, E. R. Manne-Nicholas ${ }^{8}$, M. Mason ${ }^{25}$, S. M. McGraw ${ }^{4,38}$, K. Mogren ${ }^{1}$, C. Montouri ${ }^{39}$, J. W. Moody ${ }^{11}$ (i),

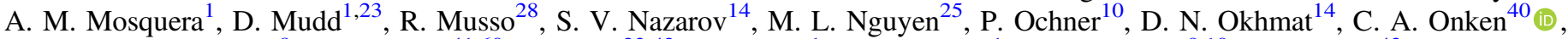
B. Ou-Yang ${ }^{8}$, A. Pancoast ${ }^{41,60}$ (1), L. Pei ${ }^{23,42}$, M. Penny ${ }^{1}$ (D), R. Poleski ${ }^{1}$ (iD, E. Portaluri ${ }^{9,10}$, J.-L. Prieto ${ }^{43}$ (1),

A. M. Price-Whelan ${ }^{19,44}$ (D) N. G. Pulatova ${ }^{14,45}$, S. Rafter ${ }^{46}$, R. M. Roettenbacher ${ }^{21,47}$ (1) , E. Romero-Colmenero ${ }^{48,49}$, J. Runnoe ${ }^{4,5,21}$, J. S. Schimoia ${ }^{1,50}$, B. J. Shappee ${ }^{1,51}$, N. Sherf ${ }^{13}$, G. V. Simonian ${ }^{1}$, A. Siviero ${ }^{9}$, D. M. Skowron ${ }^{1,52}$ (1), J. Skowron ${ }^{1,52}$, G. Somers ${ }^{1,53,61}$ (1) , M. Spencer ${ }^{11}$, D. A. Starkey ${ }^{7}$, D. J. Stevens ${ }^{1}$ (i), R. Stoll ${ }^{1}$, E. Tamajo ${ }^{53}$, J. Tayar ${ }^{1}$ (i), J. L. van Saders ${ }^{1,51}$, S. Valenti ${ }^{54}$ (10, S. Villanueva, Jr. ${ }^{1}$, C. Villforth ${ }^{7,55}$ (i), Y. Weiss ${ }^{13}$, H. Winkler $^{56}$ (D), J. Zastrow ${ }^{21}$, W. Zhu ${ }^{1}$, and Y. Zu ${ }^{1,2,57}$

${ }^{1}$ Department of Astronomy, The Ohio State University, $140 \mathrm{~W} 18$ th Ave, Columbus, OH 43210, USA

${ }^{2}$ Center for Cosmology \& AstroParticle Physics, The Ohio State University, 191 West Woodruff Ave, Columbus, OH 43210, USA

${ }^{3}$ Space Telescope Science Institute, 3700 San Martin Drive, Baltimore, MD 21218, USA

${ }^{4}$ Department of Astronomy and Astrophysics, Eberly College of Science, The Pennsylvania State University, 525 Davey Laboratory, University Park, PA 16802, USA

${ }^{5}$ Institute for Gravitation and the Cosmos, The Pennsylvania State University, University Park, PA 16802, USA

${ }_{7}^{6}$ Illumination Works, LLC, 5650 Blazer Parkway, Dublin, OH 43017, USA

${ }^{7}$ SUPA Physics and Astronomy, University of St. Andrews, KY16 9SS, UK

${ }^{8}$ Department of Physics and Astronomy, Georgia State University, 25 Park Place, Suite 605, Atlanta, GA 30303, USA

${ }^{9}$ Dipartimento di Fisica e Astronomia "G. Galilei," Università di Padova, Vicolo dell'Osservatorio 3, I-35122 Padova, Italy ${ }^{10}$ INAF-Osservatorio Astronomico di Padova, Vicolo dell'Osservatorio 5 I-35122, Padova, Italy

${ }^{11}$ Department of Physics and Astronomy, N283 ESC, Brigham Young University, Provo, UT 84602, USA

${ }^{12}$ Wise Observatory and School of Physics and Astronomy, Raymond and Beverly Sackler Faculty of Exact Sciences, Tel Aviv University, Tel Aviv 69978, Israel

${ }^{14}$ Crimean Astrophysical Observatory, P/O Nauchny, Crimea 298409, Russia ${ }^{\dagger}$

${ }^{15}$ Dark Cosmology Centre, Niels Bohr Institute, University of Copenhagen, Juliane Maries Vej 30, DK-2100 Copenhagen, Denmark

${ }^{16}$ Steward Observatory, University of Arizona, 933 North Cherry Avenue, Tucson, AZ 85721, USA

${ }^{17}$ Cahill Center for Astrophysics, California Institute of Technology, Pasadena, CA 91125, USA

${ }^{18}$ Google, Inc., 1600 Amphitheatre Pkwy, Bldg 40, Mountain View, CA, 94043, USA

${ }^{19}$ Department of Astronomy, Columbia University, 550 West 120th Street, New York, NY 10027, USA

${ }^{20}$ Department of Physics, University of California at San Diego, La Jolla, CA 92037, USA
${ }^{21}$ Department of Astronomy, University of Michigan, 1085 S. University Avenue, Ann Arbor, MI 48109, USA

22 Department of Terrestrial Magnetism, Carnegie Institution of Washington, 5241 Broad Branch Road NW, Washington, DC 20015-1305, USA

${ }^{23}$ Department of Physics and Astronomy, 4129 Frederick Reines Hall, University of California, Irvine, CA 92697, USA

${ }^{24}$ Center for Exoplanets and Habitable Worlds, The Pennsylvania State University, University Park, PA 16802, USA

${ }^{25}$ Department of Physics and Astronomy, University of Wyoming, 1000 E. University Ave. Laramie, WY 82071, USA

${ }^{26}$ Department of Biology, Geology, and Physical Sciences, Sul Ross State University, WSB 216, Box-64, Alpine, TX, 79832, USA

${ }^{27}$ Las Cumbres Observatory, 6740 Cortona Drive, Suite 102, Goleta, CA 93117, USA

${ }^{28}$ Fountainwood Observatory, Department of Physics FJS 149, Southwestern University, 1011 E. University Ave., Georgetown, TX 78626, USA

${ }^{29}$ Department of Physics and Astronomy, University of Missouri, Columbia, MO 65211, USA

30 Jet Propulsion Laboratory, California Institute of Technology, 4800 Oak Grove Drive, Pasadena, CA 91109, USA

${ }^{31}$ Space Science and Astrobiology Division, NASA Ames Research Center, M/S 244-30, Moffett Field, CA 94035, USA

${ }^{32}$ Physics Department, CUNY Graduate Center, New York, NY 10016, USA

${ }_{33}$ Department of Physics and Astronomy, MS 4242, Texas A\&M University, College Station, TX 77843-4242, USA

${ }^{34}$ Department of Physics and Astronomy, Dartmouth College, 6127 Wilder Laboratory, Hanover, NH 03755, USA

${ }^{35}$ Institute of Astronomy, National Tsing Hua University, No. 101, Section 2, Kuang-Fu Road, Hsinchu 30013, Taiwan, Republic of China

${ }^{36}$ School of Physics, University of Melbourne, Parkville, VIC 3010, Australia
${ }^{37}$ Department of Physics and Astronomy, Western Kentucky University, 1906 College Heights Blvd \#11077, Bowling Green, KY 42101, USA

38 Department of Physics \& Astronomy, Ohio University, Athens, OH 45701, USA

39 DiSAT, Universita dell'Insubria, via Valleggio 11, I-22100, Como, Italy

${ }^{40}$ Research School of Astronomy and Astrophysics, Australian National University, Canberra, ACT 2611, Australia

${ }^{41}$ Harvard-Smithsonian Center for Astrophysics, 60 Garden Street, Cambridge, MA 02138, USA

${ }^{42}$ Department of Astronomy, University of Illinois at Urbana-Champaign, Urbana, IL 61801, USA

${ }^{43}$ Núcleo de Astronomía, Facultad de Ingeniería, Universidad Diego Portales, Ejército 441 Santiago, Chile

${ }^{44}$ Department of Astrophysical Sciences, Princeton University, 4 Ivy Lane, Princeton, NJ 08544, USA

${ }^{45}$ Main Astronomical Observatory, Astro/Space Information and Computing Centre, 27 Akademika Zabolotnoho St., Kyiv 03680, Ukraine

${ }^{46}$ Department of Physics, Faculty of Natural Sciences, University of Haifa, Haifa 31905, Israel

${ }^{47}$ Department of Astronomy, AlbaNova University Center, Stockholm University, SE-106 91 Stockholm, Sweden

${ }^{48}$ South African Astronomical Observatory, P.O. Box 9, Observatory 7935, Cape Town, South Africa

${ }^{49}$ Southern African Large Telescope Foundation, P.O. Box 9, Observatory 7935, Cape Town, South Africa

${ }^{50}$ Instituto de Física, Universidade Federal do Rio do Sul, Campus do Vale, Porto Alegre, Brazil 


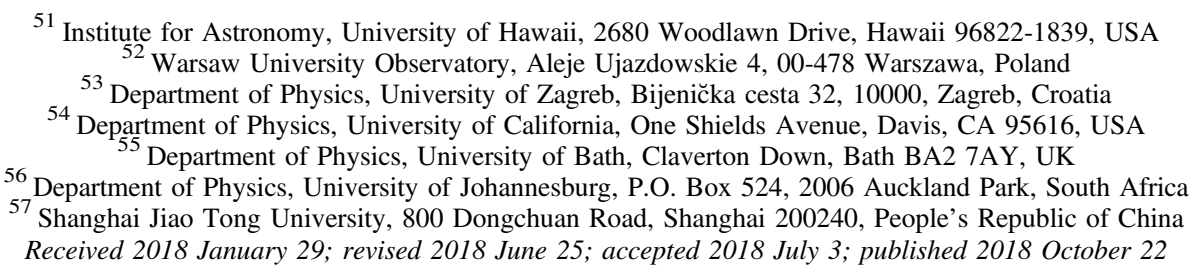

\begin{abstract}
We present the first results from a reverberation-mapping campaign undertaken during the first half of 2012, with additional data on one active galactic nucleus (AGN) (NGC 3227) from a 2014 campaign. Our main goals are (1) to determine the black hole masses from continuum- $\mathrm{H} \beta$ reverberation signatures, and (2) to look for velocitydependent time delays that might be indicators of the gross kinematics of the broad-line region. We successfully measure $\mathrm{H} \beta$ time delays and black hole masses for five AGNs, four of which have previous reverberation mass measurements. The values measured here are in agreement with earlier estimates, though there is some intrinsic scatter beyond the formal measurement errors. We observe velocity-dependent $\mathrm{H} \beta$ lags in each case, and find that the patterns have changed in the intervening five years for three AGNs that were also observed in 2007.
\end{abstract}

Key words: galaxies: active - galaxies: nuclei - galaxies: Seyfert

Supporting material: machine-readable tables

\section{Introduction}

Variability of the broad emission-line fluxes and profiles is commonly seen in the spectra of Type 1 active galactic nuclei (AGNs). A number of isolated cases of dramatic emission-line changes were reported based on photographic spectrograms by the late 1960s and early 1970s (e.g., Andrillat 1968; Pastoriza \& Gerola 1970; see the reviews by Pronik 1980 and CollinSouffrin 1980). Additional and more convincing instances of emission-line changes were found on surprisingly short timescales with the advent of linear detectors for spectrometers on ground-based telescopes (e.g., Tohline \& Osterbrock 1976; Boksenberg \& Netzer 1977; Foltz et al. 1981; Kollatschny et al. 1981; Schulz \& Rafanelli 1981; Peterson et al. 1982; Antonucci \& Cohen 1983) and in the UV with the International Ultraviolet Explorer (e.g., Ulrich et al. 1984). The interested reader is referred to Peterson (1988) for a review of the early studies of emission-line variability in AGNs.

That correlated variability of continuum and emission-line fluxes could be used to probe the structure of the broad-line region (BLR) in AGNs was recognized in the first decade of quasar research (Bahcall et al. 1972). The concept was refined in the early 1980s and has been known since as "reverberation mapping" (Blandford \& McKee 1982) because the emission lines "reverberate" in response to continuum variations. Reverberation mapping has since become a standard tool for studying the structure and dynamics of the BLR (Peterson 1993, 2014). Many programs were undertaken in the 1990s, largely enabled by the proliferation of high-quality detectors on small- to medium-sized telescopes where groups of observers could obtain enough telescope time for long-term monitoring campaigns.

In its simplest form, reverberation mapping is used to measure the mean response time $\tau$ of emission lines to

\footnotetext{
58 NSF Postdoctoral Research Fellow.

59 Rubin Fellow.

60 Einstein Fellow.

61 VIDA Postdoctoral Fellow.

$\dagger$ While the AAS journals adhere to and respect UN resolutions regarding the designations of territories (available at http://www.un.org/press/en), it is our policy to use the affiliations provided by our authors on published articles.
}

continuum variations, and this is interpreted as the light traveltime across the BLR radius $R=c \tau$. By combining the measured time delay, or lag, between continuum and emission-line flux variations with some suitable measure of the emission-line width $\Delta V$, it is possible to estimate the mass of the supermassive black hole that is the central engine of the AGN. The mass is usually expressed as

$$
M_{\mathrm{BH}}=f\left(\frac{c \tau \Delta V^{2}}{G}\right),
$$

where $G$ is the gravitational constant and $f$ is an unknown scaling constant. The quantity in parentheses is often referred to as the "virial product" (VP), which has units of mass and contains only the two observables $(\tau$ and $\Delta V$ ) and physical constants. All complicating factors, such as the inclination of the system or the effects of anisotropic line emission, are subsumed into the constant $f$. Thus, $f$ is expected to be different for every individual AGN, but should be approximately constant for every emission line in a given AGN assuming similar geometries and dynamics of the line-emitting gas. In every case where the lags from multiple emission lines can be measured in a single object, it is found that $\tau \propto \Delta V^{-2}$, as expected from Equation (1), suggesting that this is the case (Peterson \& Wandel 1999, 2000; Kollatschny 2003; Peterson et al. 2004; Bentz et al. 2010b). It is worth reminding the reader that the scale factor $f$ depends on which parameter is used to characterize the emission-line width, as we discuss in Section 4.

The scaling factor $f$ can be determined for an AGN if there is an independent measurement of the black hole mass. Unfortunately, there are few cases where the black hole radius of influence is large enough that either stellar or gas dynamical modeling can also be used. At the present time, there are stellar dynamical masses (Davies et al. 2006; Onken et al. 2014) and gas dynamical masses (Hicks \& Malkan 2008) for NGC 3227 and NGC 4151; these are useful for comparison purposes, but it would be unwise to attempt to calibrate the entire reverberation-based mass scale on only two objects. Instead, one of the well-known correlations between central black hole mass and properties of the host galaxies can be used. The first of these 

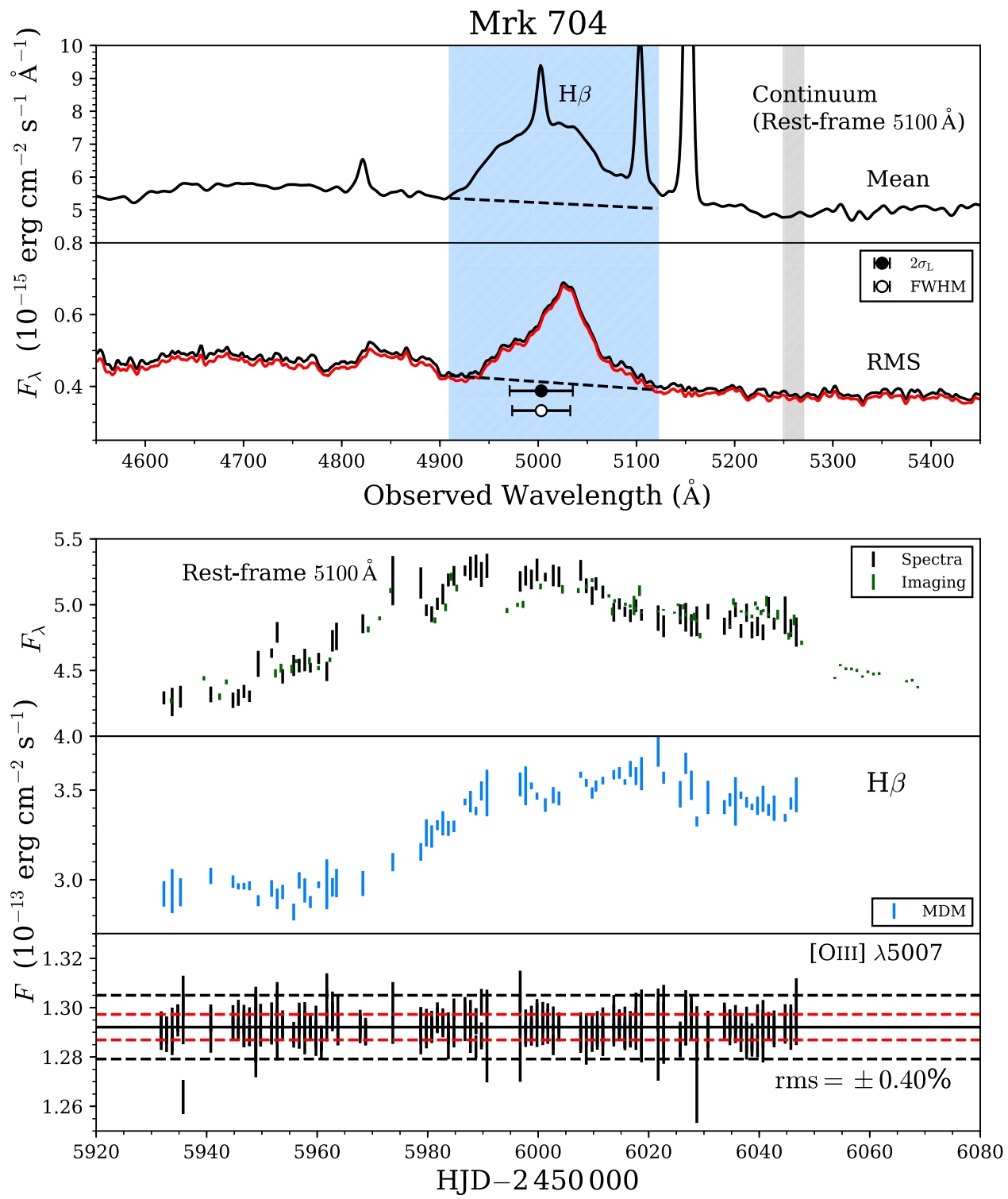

Figure 1. Top panel: weighted mean spectrum $\langle F(\lambda)\rangle$ (Equation (2)) of Mrk 704 in the observed frame based on the MDM spectra. The blue shaded region shows the integration range for $\mathrm{H} \beta$ and the dashed line underneath shows the underlying continuum assumed in the line integration. The $5100 \AA$ continuum measurement is the average flux in the gray-shaded region. Second panel: rms spectrum $\sigma_{\mathrm{rms}}(\lambda)$ (Equation (3)) in black, and the intrinsic variability $\sigma_{\mathrm{var}}(\lambda)($ Equation (4)) in red. The errorbars show the rms linewidth $\left(\sigma_{\mathrm{L}}\right)$ and full-width at half maximum. We note that He II $\lambda 4686$ also appears in the rms residual spectrum; a more sophisticated analysis will be required to separate the He II emission from blended Fe II emission and features in the host-galaxy spectrum. Lower three panels, from top to bottom: light curves for the $5100 \AA$ continuum, $\mathrm{H} \beta$ emission line, and [O III] $\lambda 5007$ narrow emission line, with the last used as a measure of the fidelity of the flux calibration. In the bottom panel, red dashed lines indicate the $1 \sigma$ scatter, while the black dashed lines indicate $\pm 1 \%$ of the mean flux.

to be used to calibrate the AGN black hole mass scale was the correlation between black hole mass and host galaxy luminosity (Magorrian et al. 1998) by Laor (1998). More recently, calibration of the AGN black hole mass scale has been based on the strong correlation between the black hole mass and the velocity dispersion of host-galaxy bulge, the $M_{\mathrm{BH}^{-}} \sigma_{*}$ relationship, which applies to both quiescent (Ferrarese \& Merritt 2000; Gebhardt et al. 2000a; Tremaine et al. 2002; Gültekin et al. 2009; McConnell et al. 2011; McConnell \& Ma 2013) and active galaxies (Gebhardt et al. 2000b; Ferrarese et al. 2001; Nelson et al. 2004; Onken et al. 2004; Dasyra et al. 2007; Woo et al. 2010, 2015; Graham et al. 2011; Park et al. 2012; Grier et al. 2013a; Batiste et al. 2017). With $\sigma_{*}$ measurements now available for $\sim 30$ AGNs from the reverberation-mapping database (Woo et al. 2015), an ensemble average $\langle f\rangle=4.47 \pm 1.25$ can be computed by comparing the predicted masses from the $M_{\mathrm{BH}}-\sigma_{*}$ relationship with the observed VPs, using the line dispersion to characterize the line width. Using this prescription, black hole masses have been measured for $\sim 60$ AGNs using reverberation mapping (see Bentz \& Katz 2015 for an up-to-date compilation).

An important result from reverberation mapping is the observed " $R-L$ " relationship between the size of the BLR and the AGN luminosity (Wandel et al. 1999; Kaspi et al. 2000, 2005; Bentz et al. 2006b, 2009a, 2013). This $R-L$ relationship allows us to bypass resource-intensive reverberation mapping by using the luminosity to infer the BLR radius. By combining the estimate of the BLR radius with the emission-line width, we can apply Equation (1) to estimate the black hole mass (see Vestergaard et al. 2011 for a review on single-epoch $M_{\mathrm{BH}}$ estimates). 

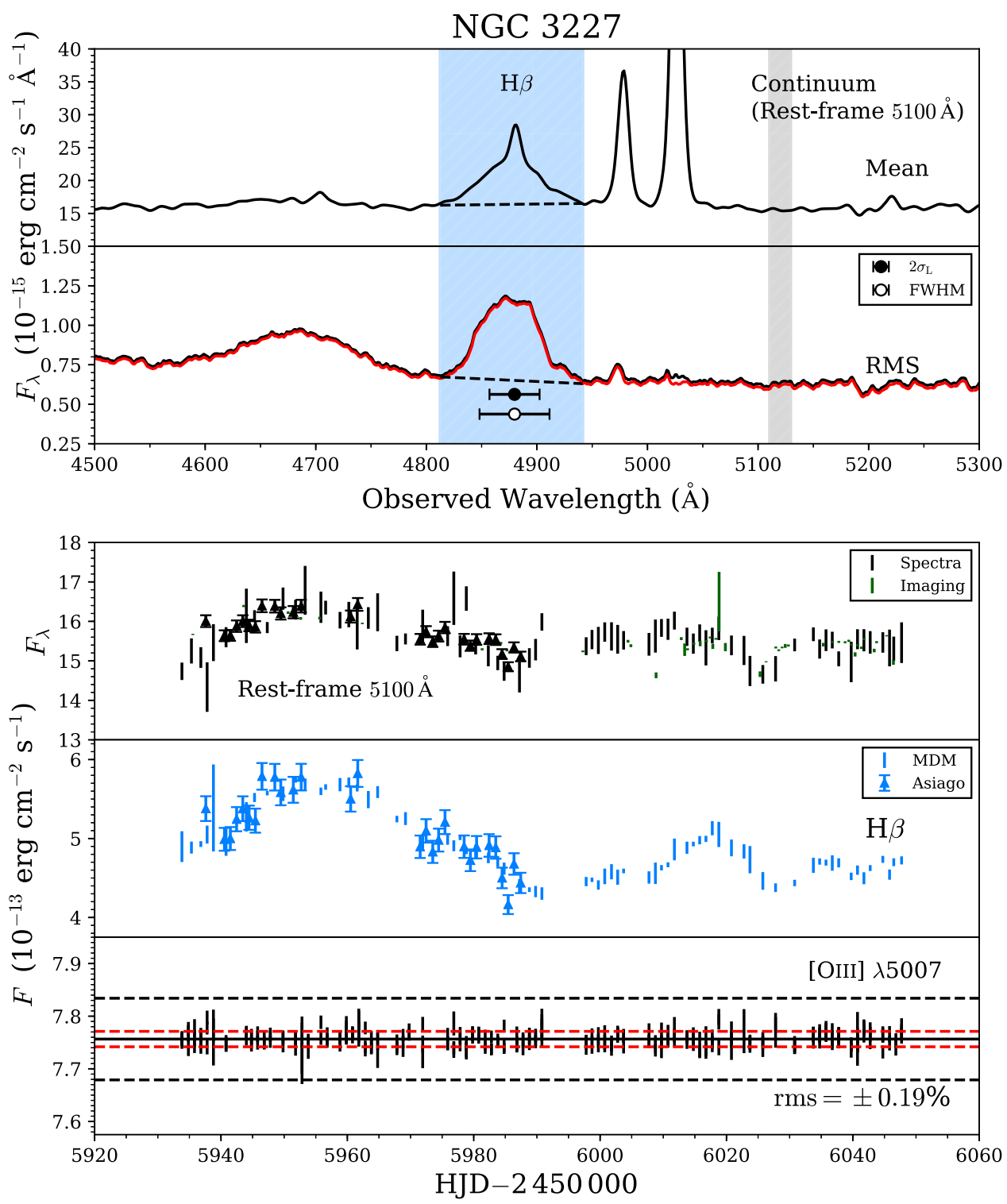

Figure 2. Mean and rms spectra for the 2012 observations of NGC 3227 and the $5100 \AA$ continuum, H $\beta$, and [O III] $\lambda 5007$ light curves. The format is the same as in Figure 1.

The frontier of reverberation mapping is determination of the kinematics and geometry of the BLR by examination of the emission-line response as a function of line-of-sight velocity. The ultimate goal is to either model the BLR geometry and kinematics directly (e.g., Pancoast et al. 2012, 2014; Waters et al. 2016) or to recover velocity-delay maps and model the BLR indirectly (e.g., Bentz et al. 2010a; Grier et al. 2013b). Observational results are only now beginning to appear as the technical requirements for detailed reverberation mapping are quite demanding (Horne et al. 2004).

Over the last decade, we have undertaken a new series of reverberation programs with several specific goals in mind.

1. To increase the number of AGNs for which reverberation lags are measured for the $\mathrm{H} \beta$ emission line. Additional data can better constrain the $R-L$ relationship (Bentz et al. 2013) and the AGN $M_{\mathrm{BH}}-\sigma_{*}$ relationship that underlies the reverberation-based black hole mass calibration scale (Grier et al. 2013a).
2. To improve upon previous reverberation results. Our reanalysis of nearly all the reverberation data that existed a decade ago revealed that many of the sources would benefit from a higher sampling rate (Peterson et al. 2004).

3. To obtain higher-quality, higher-time-resolution spectra that would enable recovery of velocity-delay maps (e.g., Grier et al. 2013b).

These programs were designed to meet the criteria described by Horne et al. (2004) to enable recovery of velocity-delay maps. They were carried out at MDM and partner observatories in 2005 (Bentz et al. 2006a, 2007; Denney et al. 2006), 2007 (Denney et al. 2009a, 2009b, 2010), and 2010 (Grier et al. 2012a, 2012b, 2013b). In addition to these ground-based programs, we carried out an intensive multiwavelength campaign on NGC 5548 known as the AGN Space Telescope and Optical Reverberation Mapping (AGN STORM) project (De Rosa et al. 2015; Edelson et al. 2015; Fausnaugh et al. 2016; Goad et al. 2016; Mathur et al. 2017; Pei et al. 2017; Starkey et al. 2017) and a concurrent optical monitoring 

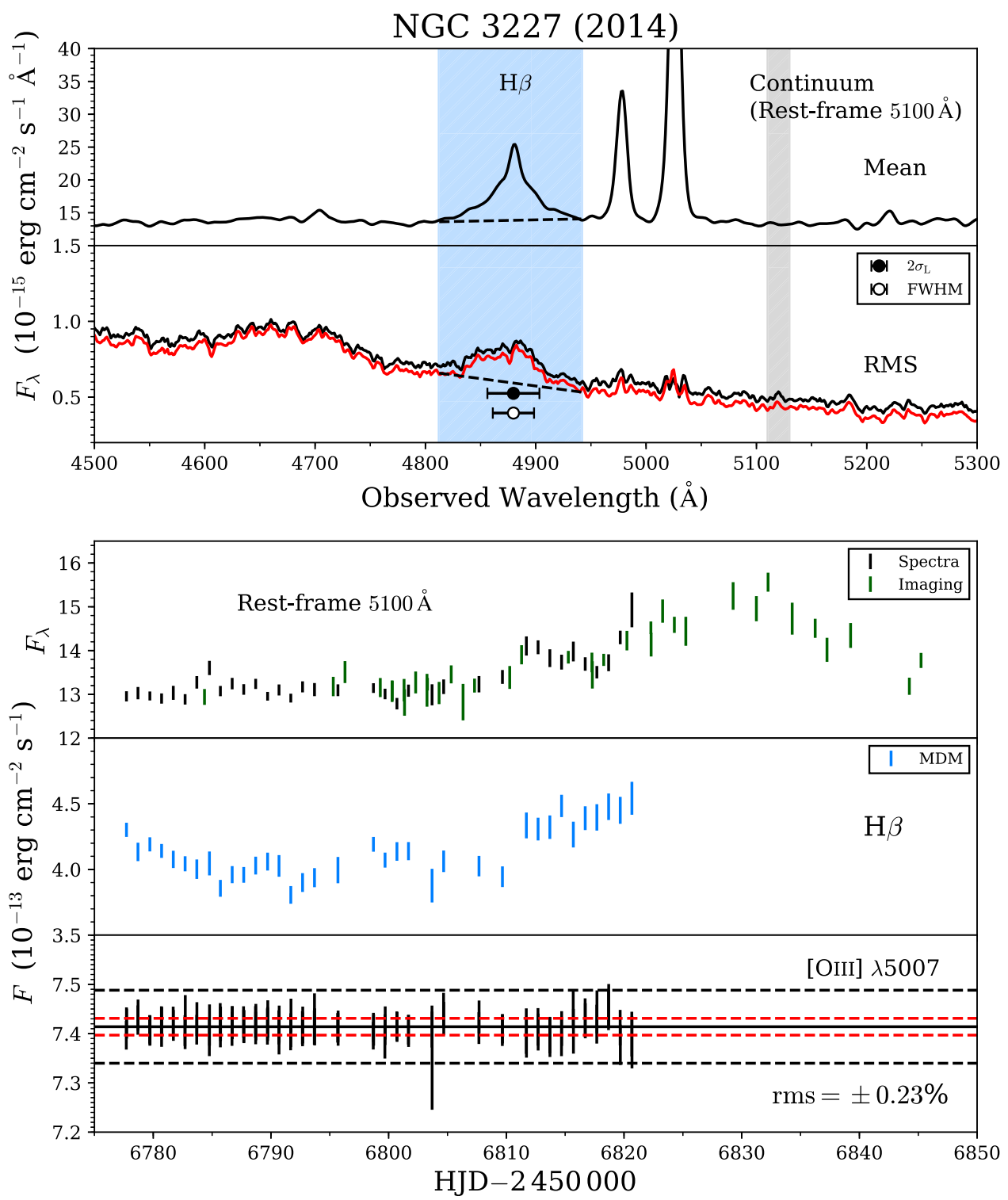

Figure 3. Mean and rms spectra for the 2014 observations of NGC 3227 and the $5100 \AA$ A continuum, $\mathrm{H} \beta$, and [O III] $\lambda 5007$ light curves. The format is the same as in Figure 1.

program on additional AGNs (Fausnaugh et al. 2017). The amount of AGN reverberation data has increased dramatically over the last few years, with several other groups carrying out campaigns similar to ours (Bentz et al. 2009b, 2010a, 2014, 2016a, 2016b; Barth et al. 2011a, 2011b, 2013, 2015; Du et al. 2014, 2015, 2016; Pei et al. 2014; Wang et al. 2014). Ongoing large multi-object reverberation-mapping campaigns (King et al. 2015; Shen et al. 2015) are expected to significantly increase the number of reverberation-mapped AGNs, as well as increase redshift and luminosity ranges of the sample, especially for emission lines other than the Balmer series.

Here we report results from a campaign undertaken in early 2012. We also include additional results on NGC 3227 from 2014. We describe the observations and data analysis in Section 2. Our time-series analysis is presented in Section 3 and our black hole mass measurement is explained in Section 4. We briefly discuss and summarize our results in Section 5. When needed, we adopt a cosmological model with $\Omega_{m}=$ $0.30, \Omega_{\Lambda}=0.70$, and $H_{0}=70 \mathrm{~km} \mathrm{~s}^{-1} \mathrm{Mpc}^{-1}$.

\section{Observations and Data Analysis}

\subsection{Target Selection}

The primary objective of this campaign is to determine the kinematics and structure of the BLR in a few well-studied bright AGNs. In particular, we are re-examining NGC 3227, NGC 3516, and NGC 5548 from Denney et al. (2009a), for which crosscorrelation of individual velocity bins suggested gross kinematics of outflow, infall, and rotation/virialization, respectively. As we discuss here and elsewhere, these results need to be checked and more thoroughly characterized. We also included in our observing program NGC 4151, for which the best reverberation data are from a weather-abbreviated campaign in 2005 (Bentz et al. 2006a). In addition to these primary targets, we added a few sources that could only be observed for part of our campaign on account of their location in the sky. Sources included in the 2012 campaign were Mrk 374, Mrk 382, Mrk 478, Mrk 618, and Mrk 704. Because of the shorter monitoring period, the failure rate for these secondary sources was high, with only Mrk 704 yielding data useful for reverberation purposes. 

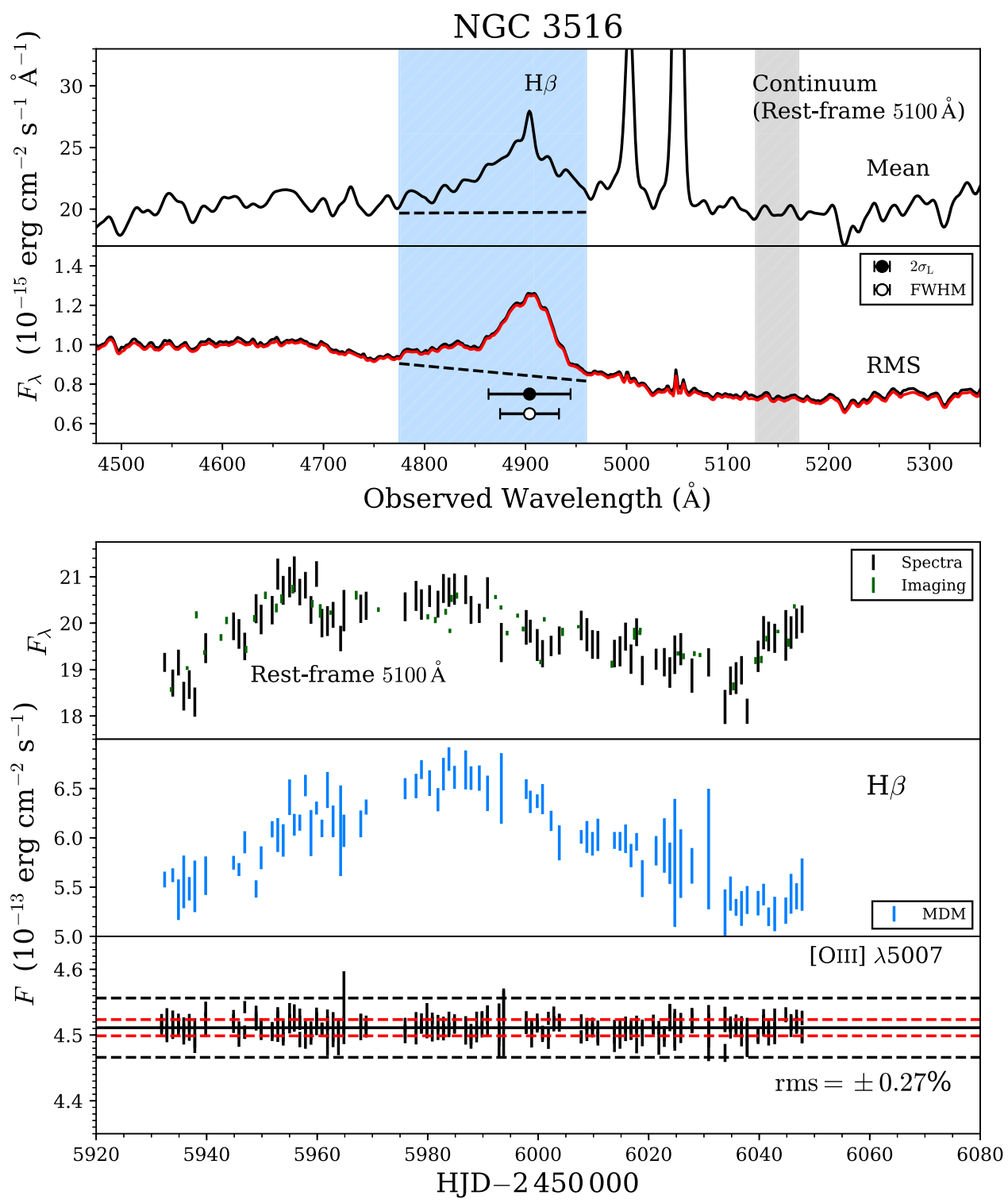

Figure 4. Mean and rms spectra for NGC 3516 and the $5100 \AA$ continuum, H $\beta$, and [O III] $\lambda 5007$ light curves. The format is the same as in Figure 1 .

The properties of the sources studied in this paper are summarized in Table 1. Both NGC 3227 and NGC 4151 are too close for redshift-based distances to be reliable. NGC 3227 is interacting with an elliptical companion, NGC 3226, which has a surface-brightness fluctuation distance of $23.5 \mathrm{Mpc}$ (Tonry et al. 2001). We therefore adopt this as the distance to NGC 3227. In the case of NGC 4151, we are currently working on a Cepheid-based distance, but here we use the distance of $13.9 \mathrm{Mpc}$ adopted by Onken et al. (2014) in their recent stellar dynamical study (although this distance is derived from Hubble's law).

\subsection{Observations}

\subsubsection{Spectroscopy}

The principal data source for both the 2012 and 2014 campaigns was the Boller and Chivens CCD spectrograph on the MDM Observatory $1.3 \mathrm{~m}$ McGraw-Hill telescope on Kitt Peak. The 2012 campaign ran from the beginning of 2012 January through the end of 2012 April. We used a $350 \mathrm{~mm}^{-1}$ grating to obtain a dispersion of $1.33 \AA$ pixel $^{-1}$. We set the grating for a central wavelength of $5150 \AA$, which resulted in spectral coverage over the range $4400-5850 \AA$. The slit was oriented north-south (position angle $\mathrm{PA}=0^{\circ}$ ) with a projected width of 5." 0 that resulted in a spectral resolution of $7.9 \AA$. We used an extraction window of 12 ". 0 along the slit.

The 2012 campaign also included spectroscopic observations obtained at the Asiago Astrophysical Observatory of Padova University with the $1.22 \mathrm{~m}$ Galileo telescope and the Cassegrain Boller \& Chivens spectrograph. We used a $300 \mathrm{~mm}^{-1}$ grating in first order combined with a 5 !" $0 \times 7 ! 75$ long slit oriented at $\mathrm{PA}=90^{\circ}$. The spectral range between about 3200 and $8000 \AA$ was covered with a dispersion of $2.3 \mathrm{~A} \mathrm{pixel}^{-1}$. The spatial scale was 1 arcsec pixel ${ }^{-1}$; the resulting resolution was $10.5 \AA$. We used an extraction window of 12 !" 0 .

The Crimean Astrophysical Observatory (CrAO) provided spectra from the Nasmith spectrograph and SPEC-10 CCD on the $2.6 \mathrm{~m}$ Shajn telescope. We used a 3!"0 slit at a position angle of $90^{\circ}$, and an extraction window of 11 !" 0 . The CrAO 

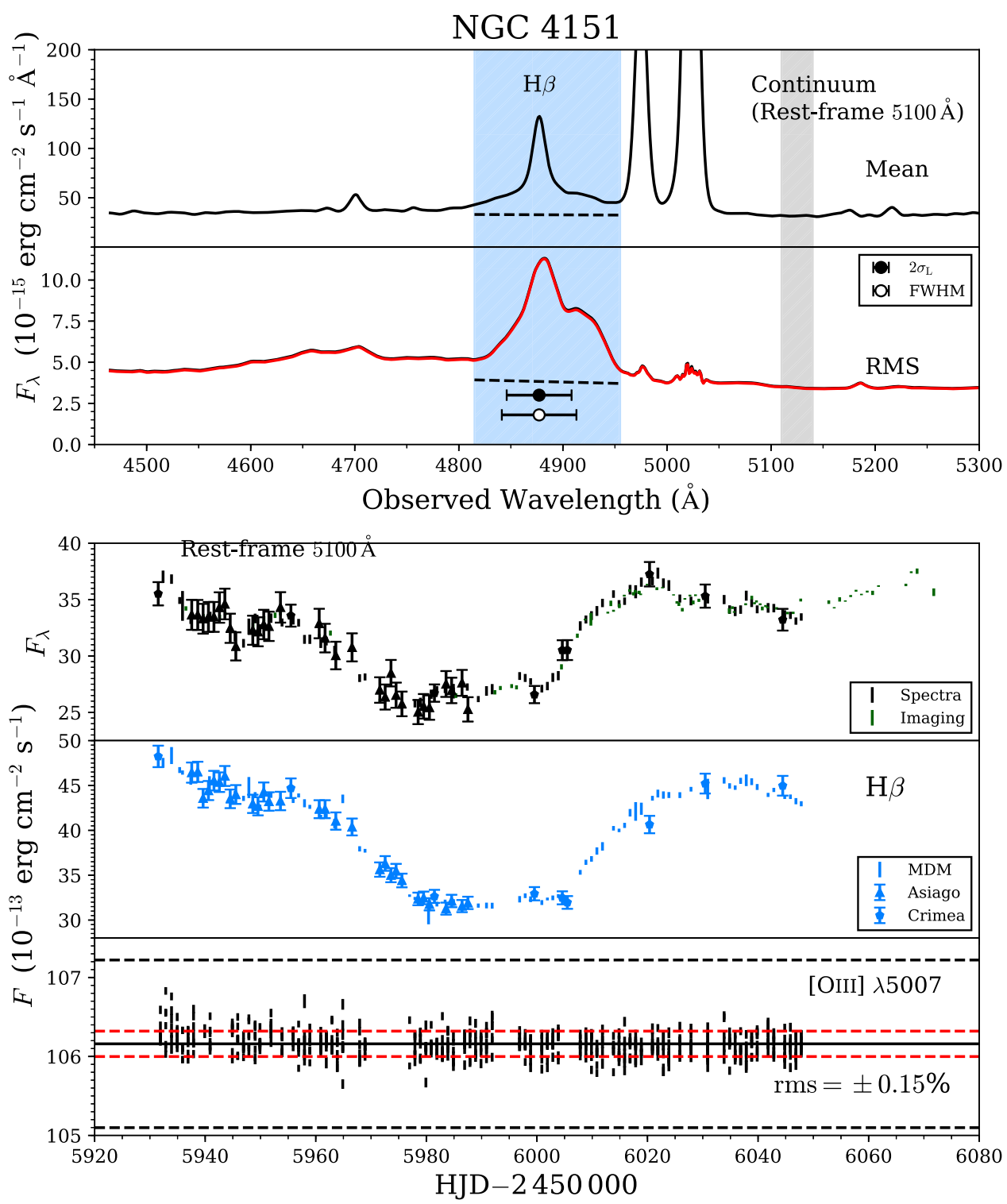

Figure 5. Mean and rms spectra for NGC 4151 and the $5100 \AA$ continuum, $\mathrm{H} \beta$, and [O III] $\lambda 5007$ light curves. The format is the same as in Figure 1 .

Table 1

Source Properties

\begin{tabular}{lccccc}
\hline \hline Object & $z$ & $\begin{array}{c}D_{L} \\
(\mathrm{Mpc}) \\
(1)\end{array}$ & $\begin{array}{c}\log \lambda L_{5100} \AA \\
\left(\mathrm{erg} \mathrm{s}^{-1}\right) \\
(4)\end{array}$ & $\begin{array}{c}\log \lambda L_{\text {host }} \\
\left(\mathrm{erg} \mathrm{s}^{-1}\right) \\
(5)\end{array}$ & $\begin{array}{c}E(B-V) \\
(\mathrm{mag}) \\
(6)\end{array}$ \\
\hline Mrk 704 & 0.0292 & 128.0 & 43.72 & 43.27 & 0.03 \\
NGC 3227 & 0.0038 & 23.5 & 42.74 & 42.48 & 0.02 \\
NGC 3516 & 0.0088 & 38.1 & 43.29 & 43.21 & 0.04 \\
NGC 4151 & 0.0033 & 13.9 & 42.61 & 42.37 & 0.02 \\
NGC 5548 & 0.0171 & 74.5 & 43.45 & 43.20 & 0.02 \\
\hline
\end{tabular}

Note. Column 2 is taken from the NASA Extragalactic Database. Column 3 gives the luminosity distance in a consensus cosmology, except for NGC 3227 and NGC 4151 as explained in the text (see Section 2.1). Column 4 gives the observed luminosity (corrected for Galactic extinction), calculated from the observed $5100 \AA$ rest-frame light curve and Column 3 and is corrected for the starlight contribution which is given in Column 5. Column 6 gives the Galactic reddening value from Schlafly \& Finkbeiner (2011).
Table 2

[O III] $\lambda 5007$ Flux Calibration

\begin{tabular}{lccc}
\hline \hline Object & $\begin{array}{c}\text { No. } \\
\text { Photometric } \\
(1)\end{array}$ & $\begin{array}{c}F([\mathrm{O} \mathrm{III}] \lambda 5007) \\
\left(10^{-13} \mathrm{erg} \mathrm{s}^{-1} \mathrm{~cm}^{-2}\right)\end{array}$ & $\begin{array}{c}\text { Percent } \\
\text { Scatter } \\
(4)\end{array}$ \\
\hline Mrk 704 & 21 & $1.31 \pm 0.03$ & 0.40 \\
NGC 3227 & 24 & $7.81 \pm 0.16$ & 0.19 \\
NGC 3516 & 21 & $4.58 \pm 0.07$ & 0.27 \\
NGC 4151 & 20 & $107 \pm 2$ & 0.15 \\
NGC 5548 & 21 & $4.91 \pm 0.08$ & 0.18 \\
\hline
\end{tabular}

Note. Column 2 gives the number of nights with clear and stable conditions and judged to be photometric. Each object had three observations per night, which were used to calculate the narrow [O III] $\lambda 5007$ line flux. The line flux and its uncertainty are given in Column 3. Column 4 gives the fractional variation of the $[\mathrm{O} \mathrm{III}] \lambda 5007$ line light curve, which serves as an estimate of the night-to-night calibration error. 

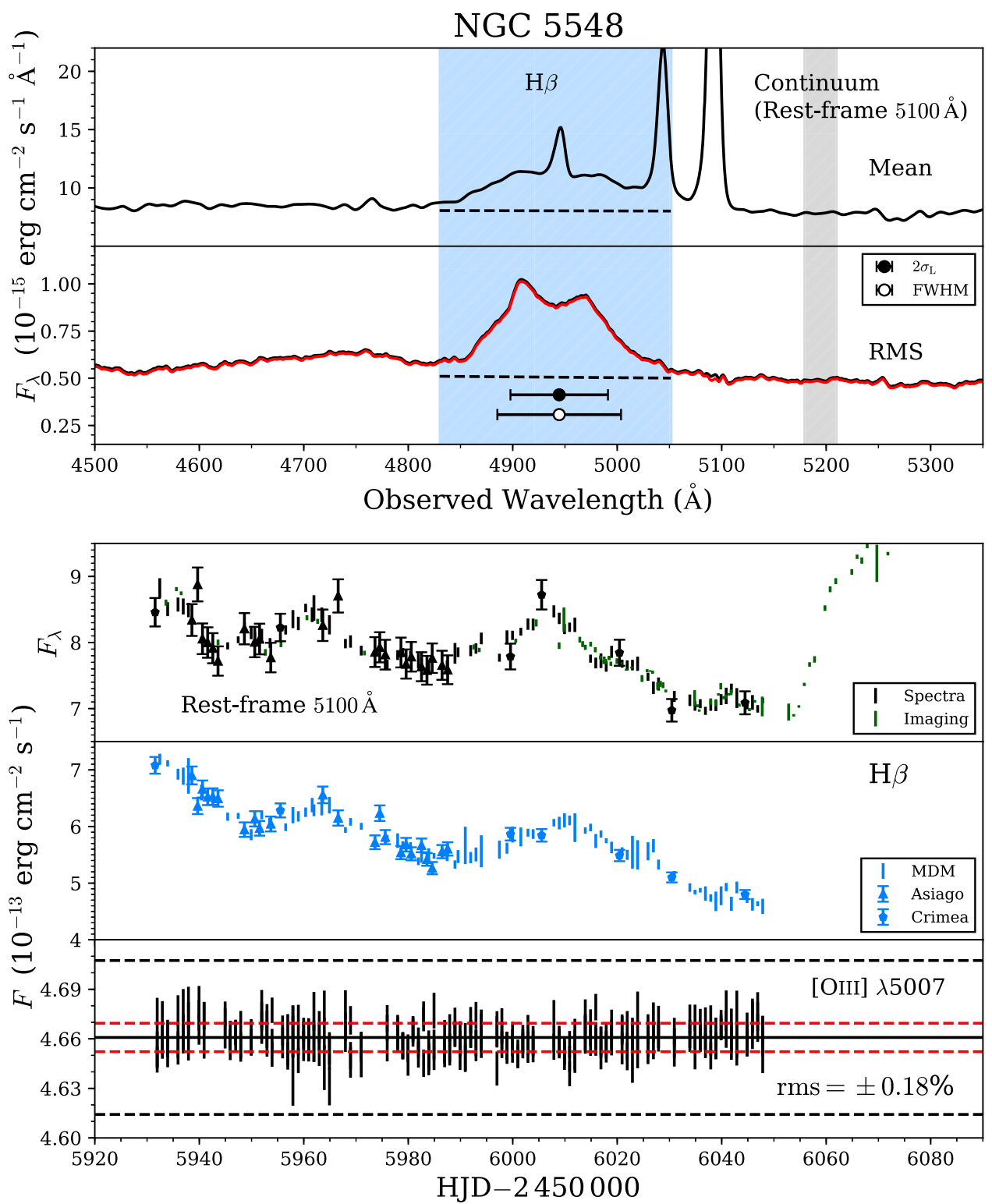

Figure 6. Mean and rms spectra for NGC 5548 and the $5100 \AA$ continuum, H $\beta$, and [O III] $\lambda 5007$ light curves. The format is the same as in Figure 1 .

Table 3

Observed-frame Integration Windows

\begin{tabular}{lccc}
\hline \hline Object & $\begin{array}{c}5100 \AA \\
(\AA)\end{array}$ & $\begin{array}{c}\mathrm{H} \beta \\
(\AA)\end{array}$ & $\begin{array}{c}{[\mathrm{O} \text { III] } \lambda 5007} \\
(\AA)\end{array}$ \\
\hline Mrk 704 & $5250-5270$ & $4910-5122$ & $5138-5168$ \\
NGC 3227 & $5110-5130$ & $4812-4942$ & $5005-5047$ \\
NGC 3516 & $5128-5170$ & $4775-4960$ & $5032-5066$ \\
NGC 4151 & $5110-5140$ & $4815-4955$ & $4998-5055$ \\
NGC 5548 & $5179-5210$ & $4830-5052$ & $5070-5110$ \\
\hline
\end{tabular}

data covered wavelengths from 3900 to $6100 \AA$, with a dispersion of $1.85 \AA$ pixel $^{-1}$. The smaller size of the slit for the $\mathrm{CrAO}$ configuration compared to the MDM and Asiago observations introduced a different amount of host galaxy light in the extracted spectra. However, the galaxy flux was not variable in time and we corrected for this in the final light curves (2.3.3).
Table 4

Observed-frame Continuum Fitting Windows

\begin{tabular}{|c|c|c|c|c|}
\hline Object & Line Side & $\begin{array}{l}\mathrm{H} \beta \\
(\AA)\end{array}$ & $\begin{array}{c}{[\mathrm{O} \text { III }]_{\circ}^{\lambda} \lambda 4959} \\
(\AA)\end{array}$ & $\begin{array}{c}{[\mathrm{O} \text { III }]_{(} \lambda 5007} \\
(\AA)\end{array}$ \\
\hline \multirow[t]{2}{*}{ Mrk 704} & Blue & $4890-4910$ & $5080-5090$ & $5128-5137$ \\
\hline & Red & $5235-5245$ & $5113-5120$ & $5169-5175$ \\
\hline \multirow[t]{2}{*}{ NGC 3227} & Blue & $4801-4811$ & $4950-4961$ & $5000-5005$ \\
\hline & Red & 4941-4950 & $4992-5002$ & $5047-5055$ \\
\hline \multirow[t]{2}{*}{ NGC 3516} & Blue & $4743-4752$ & $4970-4980$ & $5022-5032$ \\
\hline & Red & $5128-5170$ & 5014-5030 & $5067-5075$ \\
\hline \multirow[t]{2}{*}{ NGC 4151} & Blue & $4510-4520$ & $4950-4955$ & 4993-4998 \\
\hline & Red & $5110-5140$ & $4990-5000$ & $5055-5070$ \\
\hline \multirow[t]{2}{*}{ NGC 5548} & Blue & $4535-4545$ & $5017-5027$ & $5060-5070$ \\
\hline & Red & $5136-5159$ & $5058-5070$ & $5110-5120$ \\
\hline
\end{tabular}

Finally, the $2.3 \mathrm{~m}$ telescope at Wyoming Infrared Observatory (WIRO) and the WIRO Long Slit Spectrograph contributed a small number of observations, to fill in planned gaps during the 
Table 5

Continuum Light Curves

\begin{tabular}{|c|c|c|c|c|c|c|c|c|c|c|c|}
\hline \multicolumn{2}{|c|}{ Mrk 704} & \multicolumn{2}{|c|}{ NGC 3227} & \multicolumn{2}{|c|}{ NGC 3227 (2014) } & \multicolumn{2}{|c|}{ NGC 3516} & \multicolumn{2}{|c|}{ NGC 4151} & \multicolumn{2}{|c|}{ NGC 5548} \\
\hline $\mathrm{HJD}^{\mathrm{a}}$ & $F_{\lambda}{ }^{\mathrm{b}}$ & $\mathrm{HJD}^{\mathrm{a}}$ & $F_{\lambda}{ }^{\mathrm{b}}$ & $\mathrm{HJD}^{\mathrm{a}}$ & $F_{\lambda}{ }^{\mathrm{b}}$ & $\mathrm{HJD}^{\mathrm{a}}$ & $F_{\lambda}{ }^{\mathrm{b}}$ & $\mathrm{HJD}^{\mathrm{a}}$ & $F_{\lambda}{ }^{\mathrm{b}}$ & $\mathrm{HJD}^{\mathrm{a}}$ & $F_{\lambda}{ }^{\mathrm{b}}$ \\
\hline 5932.26 & $4.29 \pm 0.05 \mathrm{M}$ & 5933.82 & $14.74 \pm 0.22 \mathrm{M}$ & 6645.61 & $10.81 \pm 0.22 \mathrm{~W} 1$ & 5932.39 & $19.16 \pm 0.20 \mathrm{M}$ & 5931.51 & $35.52 \pm 1.03 \mathrm{C}$ & 5931.55 & $8.46 \pm 0.22 \mathrm{C}$ \\
\hline 5933.57 & $4.27 \pm 0.02 \mathrm{~W} 1$ & 5935.32 & $15.25 \pm 0.32 \mathrm{M}$ & 6646.60 & $10.89 \pm 0.23 \mathrm{~W} 1$ & 5933.59 & $18.57 \pm 0.05 \mathrm{~W} 1$ & 5932.38 & $37.03 \pm 0.55 \mathrm{M}$ & 5932.44 & $8.83 \pm 0.15 \mathrm{M}$ \\
\hline 5933.75 & $4.26 \pm 0.11 \mathrm{M}$ & 5935.55 & $15.67 \pm 0.02 \mathrm{~W} 1$ & 6647.62 & $11.16 \pm 0.20 \mathrm{~W} 1$ & 5933.86 & $18.71 \pm 0.29 \mathrm{M}$ & 5933.91 & $36.82 \pm 0.42 \mathrm{M}$ & 5933.62 & $8.60 \pm 0.05 \mathrm{~W} 1$ \\
\hline 5935.26 & $4.30 \pm 0.08 \mathrm{M}$ & 5936.81 & $15.08 \pm 0.26 \mathrm{M}$ & 6648.59 & $10.98 \pm 0.14 \mathrm{~W} 1$ & 5934.89 & $19.15 \pm 0.28 \mathrm{M}$ & 5935.38 & $34.92 \pm 0.30 \mathrm{M}$ & 5933.94 & $8.50 \pm 0.10 \mathrm{M}$ \\
\hline 5939.50 & $4.44 \pm 0.02 \mathrm{~W} 1$ & 5937.60 & $16.01 \pm 0.15 \mathrm{~A} 1$ & 6650.51 & $11.06 \pm 0.16 \mathrm{~W} 1$ & 5935.85 & $18.43 \pm 0.31 \mathrm{M}$ & 5935.90 & $34.44 \pm 1.32 \mathrm{M}$ & 5935.62 & $8.81 \pm 0.03 \mathrm{~W} 1$ \\
\hline 5940.75 & $4.32 \pm 0.06 \mathrm{M}$ & 5937.80 & $14.34 \pm 0.63 \mathrm{M}$ & 6653.59 & $12.52 \pm 0.34 \mathrm{~W} 1$ & 5936.47 & $19.03 \pm 0.04 \mathrm{~W} 1$ & 5936.51 & $34.22 \pm 0.19 \mathrm{~W} 1$ & 5935.93 & $8.57 \pm 0.11 \mathrm{M}$ \\
\hline 5942.35 & $4.30 \pm 0.03 \mathrm{~W} 1$ & 5938.80 & $15.46 \pm 0.47 \mathrm{M}$ & 6655.50 & $13.01 \pm 0.37 \mathrm{~W} 1$ & 5936.86 & $18.68 \pm 0.31 \mathrm{M}$ & 5937.40 & $33.10 \pm 0.26 \mathrm{M}$ & 5936.54 & $8.75 \pm 0.03 \mathrm{~W} 1$ \\
\hline 5943.56 & $4.41 \pm 0.02 \mathrm{~W} 1$ & 5940.55 & $15.64 \pm 0.14 \mathrm{~A} 1$ & 6656.51 & $13.06 \pm 0.37 \mathrm{~W} 1$ & 5937.85 & $18.30 \pm 0.31 \mathrm{M}$ & 5937.56 & $33.65 \pm 1.33 \mathrm{~A} 1$ & 5936.93 & $8.55 \pm 0.12 \mathrm{M}$ \\
\hline 5944.76 & $4.27 \pm 0.06 \mathrm{M}$ & 5940.80 & $15.60 \pm 0.25 \mathrm{M}$ & 6661.93 & $13.70 \pm 0.16 \mathrm{M}$ & 5938.14 & $20.18 \pm 0.08 \mathrm{~A} 2$ & 5938.65 & $33.64 \pm 1.33 \mathrm{~A} 1$ & 5937.93 & $8.49 \pm 0.11 \mathrm{M}$ \\
\hline 5945.74 & $4.30 \pm 0.06 \mathrm{M}$ & 5941.48 & $15.65 \pm 0.14 \mathrm{~A} 1$ & 6662.48 & $13.07 \pm 0.26 \mathrm{~W} 1$ & 5939.63 & $19.37 \pm 0.05 \mathrm{~W} 1$ & 5939.62 & $33.30 \pm 1.32 \mathrm{~A} 1$ & 5938.59 & $8.34 \pm 0.24 \mathrm{~A} 1$ \\
\hline 5946.75 & $4.34 \pm 0.05 \mathrm{M}$ & 5942.47 & $15.88 \pm 0.15 \mathrm{~A} 1$ & 6663.50 & $13.00 \pm 0.41 \mathrm{~W} 1$ & 5939.82 & $19.46 \pm 0.32 \mathrm{M}$ & 5939.65 & $33.52 \pm 0.21 \mathrm{~W} 1$ & 5939.69 & $8.88 \pm 0.26 \mathrm{~A} 1$ \\
\hline 5947.75 & $4.30 \pm 0.04 \mathrm{M}$ & 5942.54 & $15.93 \pm 0.02 \mathrm{~W} 1$ & 6663.90 & $13.75 \pm 0.13 \mathrm{M}$ & 5942.59 & $19.69 \pm 0.07 \mathrm{~W} 1$ & 5940.59 & $33.46 \pm 1.32 \mathrm{~A} 1$ & 5940.43 & $8.03 \pm 0.05 \mathrm{M}$ \\
\hline 5949.33 & $4.55 \pm 0.10 \mathrm{M}$ & 5943.45 & $16.01 \pm 0.15 \mathrm{~A} 1$ & 6664.88 & $13.94 \pm 0.14 \mathrm{M}$ & 5943.60 & $20.06 \pm 0.08 \mathrm{~W} 1$ & 5940.89 & $33.57 \pm 0.35 \mathrm{M}$ & 5940.61 & $8.06 \pm 0.23 \mathrm{~A} 1$ \\
\hline 5951.75 & $4.63 \pm 0.04 \mathrm{M}$ & 5943.60 & $16.39 \pm 0.03 \mathrm{~W} 1$ & 6665.42 & $14.26 \pm 0.21 \mathrm{~W} 1$ & 5944.87 & $19.93 \pm 0.30 \mathrm{M}$ & 5941.58 & $33.46 \pm 1.32 \mathrm{~A} 1$ & 5941.60 & $8.00 \pm 0.23 \mathrm{~A} 1$ \\
\hline 5952.45 & $4.48 \pm 0.03 \mathrm{~W} 1$ & 5944.01 & $16.14 \pm 0.69 \mathrm{M}$ & 6666.93 & $14.02 \pm 0.17 \mathrm{M}$ & 5945.84 & $19.77 \pm 0.32 \mathrm{M}$ & 5942.57 & $34.30 \pm 1.35 \mathrm{~A} 1$ & 5942.59 & $7.91 \pm 0.23 \mathrm{~A} 1$ \\
\hline 5952.76 & $4.79 \pm 0.08 \mathrm{M}$ & 5944.44 & $15.90 \pm 0.15 \mathrm{~A} 1$ & 6666.94 & $13.95 \pm 0.14 \mathrm{M}$ & 5946.89 & $19.50 \pm 0.30 \mathrm{M}$ & 5942.62 & $33.43 \pm 0.23 \mathrm{~W} 1$ & 5942.62 & $7.92 \pm 0.04 \mathrm{~W} 1$ \\
\hline 5953.45 & $4.52 \pm 0.03 \mathrm{~W} 1$ & 5945.31 & $16.14 \pm 0.14 \mathrm{M}$ & 6670.45 & $13.35 \pm 0.17 \mathrm{~W} 1$ & 5947.11 & $19.43 \pm 0.08 \mathrm{~A} 2$ & 5943.56 & $34.61 \pm 1.36 \mathrm{~A} 1$ & 5943.58 & $7.72 \pm 0.22 \mathrm{~A} 1$ \\
\hline 5953.75 & $4.46 \pm 0.05 \mathrm{M}$ & 5945.44 & $15.86 \pm 0.14 \mathrm{~A} 1$ & 6671.63 & $13.66 \pm 0.21 \mathrm{C}$ & 5948.65 & $20.10 \pm 0.07 \mathrm{~W} 1$ & 5943.62 & $33.74 \pm 0.17 \mathrm{~W} 1$ & 5943.59 & $8.00 \pm 0.04 \mathrm{~W} 1$ \\
\hline 5955.40 & $4.51 \pm 0.03 \mathrm{~W} 1$ & 5946.49 & $16.39 \pm 0.16 \mathrm{~A} 1$ & 6671.89 & $12.96 \pm 0.30 \mathrm{FWO}$ & 5948.94 & $20.31 \pm 0.31 \mathrm{M}$ & 5944.53 & $32.46 \pm 1.29 \mathrm{~A} 1$ & 5945.43 & $7.95 \pm 0.05 \mathrm{M}$ \\
\hline 5955.75 & $4.54 \pm 0.08 \mathrm{M}$ & 5947.33 & $15.84 \pm 0.14 \mathrm{M}$ & 6672.45 & $13.09 \pm 0.17 \mathrm{~W} 1$ & 5949.86 & $20.08 \pm 0.32 \mathrm{M}$ & 5945.40 & $31.77 \pm 0.48 \mathrm{M}$ & 5947.45 & $8.04 \pm 0.05 \mathrm{M}$ \\
\hline 5956.24 & $4.57 \pm 0.01 \mathrm{~W} 1$ & 5948.52 & $16.38 \pm 0.16 \mathrm{~A} 1$ & 6672.91 & $13.19 \pm 0.28 \mathrm{FWO}$ & 5950.63 & $20.60 \pm 0.08 \mathrm{~W} 1$ & 5945.54 & $30.85 \pm 1.25 \mathrm{~A} 1$ & 5948.64 & $8.21 \pm 0.24 \mathrm{~A} 1$ \\
\hline 5956.75 & $4.54 \pm 0.05 \mathrm{M}$ & 5948.65 & $16.36 \pm 0.03 \mathrm{~W} 1$ & 6674.00 & $13.27 \pm 0.27 \mathrm{FWO}$ & 5951.86 & $20.28 \pm 0.31 \mathrm{M}$ & 5946.93 & $31.13 \pm 0.38 \mathrm{M}$ & 5948.65 & $8.26 \pm 0.04 \mathrm{~W} 1$ \\
\hline 5957.76 & $4.58 \pm 0.09 \mathrm{M}$ & 5949.46 & $16.20 \pm 0.15 \mathrm{~A} 1$ & 6679.44 & $13.44 \pm 0.13 \mathrm{~W} 1$ & 5952.61 & $20.33 \pm 0.09 \mathrm{~W} 1$ & 5947.90 & $32.61 \pm 0.71 \mathrm{M}$ & 5949.94 & $8.14 \pm 0.09 \mathrm{M}$ \\
\hline 5958.60 & $4.57 \pm 0.03 \mathrm{~W} 1$ & 5949.82 & $16.58 \pm 0.28 \mathrm{M}$ & 6679.91 & $13.83 \pm 0.32 \mathrm{FWO}$ & 5952.87 & $21.06 \pm 0.33 \mathrm{M}$ & 5948.61 & $32.28 \pm 1.29 \mathrm{~A} 1$ & 5950.62 & $8.01 \pm 0.23 \mathrm{~A} 1$ \\
\hline 5958.77 & $4.52 \pm 0.04 \mathrm{M}$ & 5950.63 & $16.22 \pm 0.03 \mathrm{~W} 1$ & 6682.40 & $14.58 \pm 0.27 \mathrm{~W} 1$ & 5953.57 & $20.52 \pm 0.10 \mathrm{~W} 1$ & 5948.65 & $32.69 \pm 0.24 \mathrm{~W} 1$ & 5950.64 & $8.11 \pm 0.05 \mathrm{~W} 1$ \\
\hline$\ldots$ & $\ldots$ & $\ldots$ & $\ldots$ & $\ldots$ & $\ldots$ & $\ldots$ & $\ldots$ & $\cdots$ & $\ldots$ & $\ldots$ & $\ldots$ \\
\hline
\end{tabular}

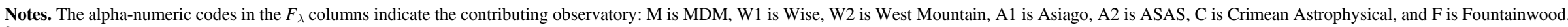

${ }^{a}$ Days - 2,450,000.

b $10^{-15} \mathrm{erg} \mathrm{s}^{-1} \mathrm{~cm}^{-2} \AA^{-1}$.

(This table is available in its entirety in machine-readable form.) 
Table 6

$\mathrm{H} \beta$ Light Curves

\begin{tabular}{|c|c|c|c|c|c|c|c|c|c|c|c|}
\hline \multicolumn{2}{|c|}{ Mrk 704} & \multicolumn{2}{|c|}{ NGC 3227} & \multicolumn{2}{|c|}{ NGC 3227 (2014) } & \multicolumn{2}{|c|}{ NGC 3516} & \multicolumn{2}{|c|}{ NGC 4151} & \multicolumn{2}{|c|}{ NGC 5548} \\
\hline$\overline{\mathrm{HJD}^{\mathrm{a}}}$ & $F^{\mathrm{b}}$ & $\mathrm{HJD}^{\mathrm{a}}$ & $F^{\mathrm{b}}$ & $\mathrm{HJD}^{\mathrm{a}}$ & $F^{\mathrm{b}}$ & $\mathrm{HJD}^{\mathrm{a}}$ & $F^{\mathrm{b}}$ & $\mathrm{HJD}^{\mathrm{a}}$ & $F^{b}$ & $\mathrm{HJD}^{\mathrm{a}}$ & $F^{\mathrm{b}}$ \\
\hline 5932.26 & $2.92 \pm 0.07 \mathrm{M}$ & 5933.82 & $4.90 \pm 0.20 \mathrm{M}$ & 6661.93 & $3.48 \pm 0.15 \mathrm{M}$ & 5932.39 & $5.58 \pm 0.08 \mathrm{M}$ & 5931.51 & $48.24 \pm 1.20 \mathrm{C}$ & 5931.55 & $7.08 \pm 0.15 \mathrm{C}$ \\
\hline 5933.75 & $2.94 \pm 0.12 \mathrm{M}$ & 5935.32 & $4.89 \pm 0.08 \mathrm{M}$ & 6663.90 & $3.61 \pm 0.05 \mathrm{M}$ & 5933.86 & $5.62 \pm 0.07 \mathrm{M}$ & 5932.38 & $47.52 \pm 0.55 \mathrm{M}$ & 5932.44 & $7.19 \pm 0.10 \mathrm{M}$ \\
\hline 5935.26 & $2.93 \pm 0.08 \mathrm{M}$ & 5936.81 & $4.93 \pm 0.04 \mathrm{M}$ & 6664.88 & $3.74 \pm 0.06 \mathrm{M}$ & 5934.89 & $5.37 \pm 0.21 \mathrm{M}$ & 5933.91 & $48.33 \pm 0.95 \mathrm{M}$ & 5933.94 & $7.12 \pm 0.05 \mathrm{M}$ \\
\hline 5940.75 & $3.02 \pm 0.05 \mathrm{M}$ & 5937.60 & $5.38 \pm 0.16 \mathrm{~A} 1$ & 6666.93 & $3.90 \pm 0.09 \mathrm{M}$ & 5935.85 & $5.55 \pm 0.27 \mathrm{M}$ & 5935.38 & $46.72 \pm 0.33 \mathrm{M}$ & 5935.93 & $6.92 \pm 0.10 \mathrm{M}$ \\
\hline 5944.76 & $2.99 \pm 0.03 \mathrm{M}$ & 5937.80 & $5.05 \pm 0.11 \mathrm{M}$ & 6666.94 & $3.97 \pm 0.09 \mathrm{M}$ & 5936.86 & $5.48 \pm 0.12 \mathrm{M}$ & 5935.90 & $46.44 \pm 0.24 \mathrm{M}$ & 5936.93 & $6.89 \pm 0.15 \mathrm{M}$ \\
\hline 5945.74 & $2.96 \pm 0.02 \mathrm{M}$ & 5938.80 & $5.39 \pm 0.55 \mathrm{M}$ & 6777.72 & $4.30 \pm 0.05 \mathrm{M}$ & 5937.85 & $5.51 \pm 0.26 \mathrm{M}$ & 5937.40 & $45.51 \pm 0.42 \mathrm{M}$ & 5937.93 & $6.89 \pm 0.32 \mathrm{M}$ \\
\hline 5946.75 & $2.96 \pm 0.02 \mathrm{M}$ & 5940.55 & $4.99 \pm 0.15 \mathrm{~A} 1$ & 6778.70 & $4.13 \pm 0.07 \mathrm{M}$ & 5939.82 & $5.62 \pm 0.20 \mathrm{M}$ & 5937.56 & $46.44 \pm 1.12 \mathrm{~A} 1$ & 5938.59 & $6.90 \pm 0.16 \mathrm{~A} 1$ \\
\hline 5947.75 & $2.97 \pm 0.02 \mathrm{M}$ & 5940.80 & $4.95 \pm 0.17 \mathrm{M}$ & 6779.71 & $4.19 \pm 0.05 \mathrm{M}$ & 5944.87 & $5.75 \pm 0.07 \mathrm{M}$ & 5938.65 & $46.52 \pm 1.13 \mathrm{~A} 1$ & 5939.69 & $6.36 \pm 0.14 \mathrm{~A} 1$ \\
\hline 5949.33 & $2.88 \pm 0.03 \mathrm{M}$ & 5941.48 & $5.00 \pm 0.15 \mathrm{~A} 1$ & 6780.71 & $4.14 \pm 0.05 \mathrm{M}$ & 5945.84 & $5.68 \pm 0.07 \mathrm{M}$ & 5939.62 & $43.57 \pm 1.04 \mathrm{~A} 1$ & 5940.43 & $6.60 \pm 0.14 \mathrm{M}$ \\
\hline 5951.75 & $2.95 \pm 0.05 \mathrm{M}$ & 5942.47 & $5.24 \pm 0.15 \mathrm{~A} 1$ & 6781.70 & $4.08 \pm 0.07 \mathrm{M}$ & 5946.89 & $5.95 \pm 0.11 \mathrm{M}$ & 5940.59 & $44.43 \pm 1.06 \mathrm{~A} 1$ & 5940.61 & $6.66 \pm 0.15 \mathrm{~A} 1$ \\
\hline 5952.76 & $2.89 \pm 0.06 \mathrm{M}$ & 5943.45 & $5.38 \pm 0.16 \mathrm{~A} 1$ & 6782.71 & $4.04 \pm 0.06 \mathrm{M}$ & 5948.94 & $5.48 \pm 0.09 \mathrm{M}$ & 5940.77 & $45.29 \pm 0.74 \mathrm{M}$ & 5941.60 & $6.52 \pm 0.15 \mathrm{~A} 1$ \\
\hline 5953.75 & $2.93 \pm 0.04 \mathrm{M}$ & 5944.01 & $5.27 \pm 0.19 \mathrm{M}$ & 6783.70 & $4.00 \pm 0.07 \mathrm{M}$ & 5949.86 & $5.80 \pm 0.12 \mathrm{M}$ & 5941.58 & $45.53 \pm 1.10 \mathrm{~A} 1$ & 5942.59 & $6.53 \pm 0.15 \mathrm{~A} 1$ \\
\hline 5955.75 & $2.82 \pm 0.05 \mathrm{M}$ & 5944.44 & $5.26 \pm 0.15 \mathrm{~A} 1$ & 6784.77 & $4.05 \pm 0.09 \mathrm{M}$ & 5951.86 & $6.09 \pm 0.08 \mathrm{M}$ & 5942.57 & $45.37 \pm 1.09 \mathrm{~A} 1$ & 5943.58 & $6.49 \pm 0.15 \mathrm{~A} 1$ \\
\hline 5956.75 & $3.00 \pm 0.05 \mathrm{M}$ & 5945.31 & $5.52 \pm 0.06 \mathrm{M}$ & 6785.70 & $3.86 \pm 0.06 \mathrm{M}$ & 5952.87 & $6.03 \pm 0.17 \mathrm{M}$ & 5943.56 & $46.06 \pm 1.11 \mathrm{~A} 1$ & 5945.43 & $6.17 \pm 0.07 \mathrm{M}$ \\
\hline 5957.76 & $2.94 \pm 0.07 \mathrm{M}$ & 5945.44 & $5.23 \pm 0.15 \mathrm{~A} 1$ & 6786.71 & $3.96 \pm 0.06 \mathrm{M}$ & 5953.85 & $5.97 \pm 0.16 \mathrm{M}$ & 5944.53 & $43.51 \pm 1.04 \mathrm{~A} 1$ & 5947.45 & $6.19 \pm 0.05 \mathrm{M}$ \\
\hline 5958.77 & $2.88 \pm 0.03 \mathrm{M}$ & 5946.49 & $5.79 \pm 0.17 \mathrm{~A} 1$ & 6787.69 & $3.96 \pm 0.06 \mathrm{M}$ & 5955.01 & $6.41 \pm 0.18 \mathrm{M}$ & 5945.40 & $44.06 \pm 0.32 \mathrm{M}$ & 5948.64 & $5.94 \pm 0.13 \mathrm{~A} 1$ \\
\hline 5960.27 & $2.97 \pm 0.02 \mathrm{M}$ & 5947.33 & $5.58 \pm 0.03 \mathrm{M}$ & 6788.70 & $4.03 \pm 0.07 \mathrm{M}$ & 5955.86 & $6.13 \pm 0.11 \mathrm{M}$ & 5945.54 & $43.99 \pm 1.05 \mathrm{~A} 1$ & 5949.94 & $5.86 \pm 0.10 \mathrm{M}$ \\
\hline 5961.75 & $2.97 \pm 0.14 \mathrm{M}$ & 5948.52 & $5.78 \pm 0.17 \mathrm{~A} 1$ & 6789.71 & $4.06 \pm 0.07 \mathrm{M}$ & 5956.86 & $6.15 \pm 0.08 \mathrm{M}$ & 5946.93 & $43.56 \pm 0.29 \mathrm{M}$ & 5950.62 & $6.14 \pm 0.13 \mathrm{~A} 1$ \\
\hline 5962.77 & $2.96 \pm 0.05 \mathrm{M}$ & 5949.46 & $5.58 \pm 0.16 \mathrm{~A} 1$ & 6790.68 & $4.03 \pm 0.08 \mathrm{M}$ & 5957.87 & $6.53 \pm 0.11 \mathrm{M}$ & 5947.90 & $44.77 \pm 1.17 \mathrm{M}$ & 5951.54 & $5.97 \pm 0.13 \mathrm{~A} 1$ \\
\hline 5963.50 & $2.98 \pm 0.08 \mathrm{M}$ & 5949.82 & $5.57 \pm 0.09 \mathrm{M}$ & 6791.69 & $3.81 \pm 0.07 \mathrm{M}$ & 5958.88 & $6.05 \pm 0.23 \mathrm{M}$ & 5948.61 & $42.95 \pm 1.02 \mathrm{~A} 1$ & 5951.95 & $6.21 \pm 0.08 \mathrm{M}$ \\
\hline 5968.27 & $2.98 \pm 0.07 \mathrm{M}$ & 5951.45 & $5.62 \pm 0.16 \mathrm{~A} 1$ & 6792.69 & $3.90 \pm 0.07 \mathrm{M}$ & 5959.88 & $6.30 \pm 0.07 \mathrm{M}$ & 5949.03 & $43.95 \pm 0.32 \mathrm{M}$ & 5953.45 & $6.08 \pm 0.07 \mathrm{M}$ \\
\hline 5973.69 & $3.10 \pm 0.05 \mathrm{M}$ & 5951.80 & $5.75 \pm 0.04 \mathrm{M}$ & 6793.70 & $3.94 \pm 0.07 \mathrm{M}$ & 5960.86 & $6.10 \pm 0.09 \mathrm{M}$ & 5949.55 & $42.68 \pm 1.01 \mathrm{~A} 1$ & 5953.69 & $6.04 \pm 0.13 \mathrm{~A} 1$ \\
\hline 5978.76 & $3.16 \pm 0.05 \mathrm{M}$ & 5952.70 & $5.78 \pm 0.17 \mathrm{~A} 1$ & 6795.69 & $4.00 \pm 0.10 \mathrm{M}$ & 5961.86 & $6.48 \pm 0.18 \mathrm{M}$ & 5949.91 & $42.63 \pm 0.23 \mathrm{M}$ & 5955.52 & $6.28 \pm 0.12 \mathrm{C}$ \\
\hline 5979.76 & $3.26 \pm 0.08 \mathrm{M}$ & 5953.32 & $5.68 \pm 0.08 \mathrm{M}$ & 6798.71 & $4.19 \pm 0.06 \mathrm{M}$ & 5962.88 & $6.17 \pm 0.16 \mathrm{M}$ & 5950.59 & $44.28 \pm 1.06 \mathrm{~A} 1$ & 5956.54 & $5.99 \pm 0.07 \mathrm{M}$ \\
\hline 5980.73 & $3.24 \pm 0.06 \mathrm{M}$ & 5955.81 & $5.60 \pm 0.05 \mathrm{M}$ & 6799.70 & $4.07 \pm 0.06 \mathrm{M}$ & 5964.24 & $6.07 \pm 0.46 \mathrm{M}$ & 5951.52 & $43.22 \pm 1.03 \mathrm{~A} 1$ & 5957.92 & $6.20 \pm 0.14 \mathrm{M}$ \\
\hline$\ldots$ & $\ldots$ & $\ldots$ & $\ldots$ & $\cdots$ & $\ldots$ & $\ldots$ & $\ldots$ & $\ldots$ & $\ldots$ & $\ldots$ & $\cdots$ \\
\hline
\end{tabular}

Notes. The alpha-numeric codes in the $F_{\lambda}$ columns indicate the contributing observatory: M is MDM, A1 is Asiago, and C is Crimean Astrophysical.

a Days - 2,450,000.

${ }^{\mathrm{b}} 10^{-13} \mathrm{erg} \mathrm{s}^{-1} \mathrm{~cm}^{-2}$

(This table is available in its entirety in machine-readable form.) 
Table 7

Light-curve Properties

\begin{tabular}{|c|c|c|c|c|c|c|c|c|c|}
\hline $\begin{array}{l}\text { Object } \\
\text { (1) }\end{array}$ & $\begin{array}{l}\text { Light Curve } \\
\text { (2) }\end{array}$ & $\begin{array}{c}N_{\text {obs }} \\
\text { (3) }\end{array}$ & $\begin{array}{c}\Delta t_{\text {med }} \\
\text { (days) } \\
(4)\end{array}$ & $\begin{array}{c}\text { Uncertainty } \\
\text { Rescaling Factor } \\
\text { (5) }\end{array}$ & $\begin{array}{l}\langle F\rangle \\
(6)\end{array}$ & $\begin{array}{c}\langle\mathrm{S} / \mathrm{N}\rangle \\
(7)\end{array}$ & $\begin{array}{l}\sigma_{\text {var }} \\
(8)\end{array}$ & $\begin{array}{c}(\mathrm{S} / \mathrm{N})_{\mathrm{var}} \\
(9)\end{array}$ & $\begin{array}{l}r_{\max } \\
(10)\end{array}$ \\
\hline \multirow{2}{*}{ Mrk 704} & $5100 \AA$ & 97 & 1.01 & 1.53 & 4.81 & 205.5 & 0.06 & 88.1 & \\
\hline & $\mathrm{H} \beta$ & 72 & 1.01 & 1.50 & 3.30 & 89.7 & 0.07 & 40.0 & $0.92 \pm 0.02$ \\
\hline \multirow[t]{2}{*}{ NGC 3227} & $5100 \AA$ & 94 & 1.01 & 2.53 & 15.60 & 175.0 & 0.02 & 27.5 & $\ldots$ \\
\hline & $\mathrm{H} \beta$ & 84 & 1.02 & 1.97 & 4.94 & 74.8 & 0.08 & 40.3 & $0.69 \pm 0.06$ \\
\hline \multirow[t]{2}{*}{ NGC 3227 (2014) } & $5100 \AA$ & 56 & 1.00 & 1.28 & 13.60 & 99.8 & 0.05 & 24.2 & $\ldots$ \\
\hline & $\mathrm{H} \beta$ & 34 & 1.00 & 1.41 & 4.14 & 55.1 & 0.04 & 9.0 & $0.77 \pm 0.05$ \\
\hline \multirow[t]{2}{*}{ NGC 3516} & $5100 \AA$ & 109 & 1.03 & 1.64 & 19.70 & 226.5 & 0.03 & 54.9 & $\ldots$ \\
\hline & $\mathrm{H} \beta$ & 77 & 1.01 & 2.54 & 5.96 & 46.8 & 0.07 & 20.1 & $0.76 \pm 0.04$ \\
\hline \multirow[t]{2}{*}{ NGC 4151} & $5100 \AA$ & 119 & 1.01 & 3.09 & 32.20 & 159.3 & 0.11 & 131.7 & $\ldots$ \\
\hline & $\mathrm{H} \beta$ & 97 & 1.01 & 3.59 & 40.10 & 111.7 & 0.13 & 103.7 & $0.94 \pm 0.01$ \\
\hline \multirow[t]{2}{*}{ NGC 5548} & $5100 \AA$ & 112 & 1.03 & 1.84 & 7.94 & 156.3 & 0.07 & 87.6 & $\ldots$ \\
\hline & $\mathrm{H} \beta$ & 91 & 1.03 & 2.61 & 5.77 & 64.2 & 0.10 & 44.7 & $0.74 \pm 0.04$ \\
\hline
\end{tabular}

Note. Column 3 gives the number of observations in each light curve. Column 4 gives the median cadence. Column 5 gives the rescaling factor by which the statistical uncertainties are multiplied to account for additional systematic errors (see Section 2.3.3). Column 6 gives the mean flux level of each light curve. The rest-frame $5100 \AA$ continuum light curves are in units of $10^{-15} \mathrm{erg} \mathrm{cm}^{-2} \mathrm{~s}^{-1} \AA^{-1}$, and the emission line light curves are in units of $10^{-13} \mathrm{erg} \mathrm{cm}^{-2} \mathrm{~s}^{-1}$. Column 7 gives the mean signal-to-noise ratio $\langle\mathrm{S} / \mathrm{N}\rangle$. Column 8 gives the rms fractional variability defined in Equation (4). Column 9 gives the approximate $\mathrm{S} / \mathrm{N}$ at which we detect variability (see Section 2.2.2). Column 10 gives the maximum value of the interpolated cross-correlation function (ICCF) (see Section 3).

Table 8

Rest-frame $\mathrm{H} \beta$ Lags

\begin{tabular}{lccc}
\hline \hline Object & $\begin{array}{c}\tau_{\text {cent }} \\
(\text { days })\end{array}$ & $\begin{array}{c}\tau_{\text {peak }} \\
(\text { days })\end{array}$ & $\begin{array}{c}\tau_{\text {JAV }} \\
(\text { days }) \\
(1)\end{array}$ \\
\hline Mrk 704 & $12)$ & $(3)$ & $14.32_{-1.06}^{+0.87}$ \\
NGC 3227 (2012) & $12.65_{-2.14}^{+1.49}$ & $14.87_{-2.45}^{+5.85}$ & $2.29_{-0.20}^{+0.23}$ \\
NGC 3227 (2014) & $1.29_{-1.27}^{+1.56}$ & $1.74_{-1.59}^{+1.69}$ & $\ldots$ \\
NGC 3516 & $2.58_{-1.31}^{+1.20}$ & $2.80_{-1.60}^{+1.0}$ & $8.27_{-0.64}^{+1.12}$ \\
NGC 4151 & $5.74_{-2.04}^{+2.26}$ & $4.24_{-3.93}^{+2.16}$ & $6.58_{-0.22}^{+0.19}$ \\
NGC 5548 & $6.82_{-0.57}^{+0.48}$ & $6.50_{-1.39}^{+0.99}$ & $3.66_{-0.52}^{+0.53}$ \\
\hline
\end{tabular}

Note. Columns 2 and 3 give the centroids and peaks, respectively, of the interpolated cross correlation functions. The uncertainties give the central 68\% confidence intervals of the cross-correlation centroid distribution (Peterson et al. 1998). Column 4 gives the lag fit by JAVELIN. The uncertainties give the central $68 \%$ confidence intervals of the JAVELIN posterior lag distributions. All lags are relative to the $5100 \AA$ continuum light curve and corrected to the rest frame.

monitoring campaign. We used a $900 \mathrm{~mm}^{-1}$ grating, resulting in a $\sim 1 \AA$ pixel $^{-1}$ dispersion between 4400 and $5600 \AA$. A 5"! 0 slit aligned at $\mathrm{PA}=0^{\circ}$ was used with a 12 !" 0 extraction window.

\subsubsection{Imaging}

We supplemented our spectroscopic continuum light curves with photometric observations. Observations in 2012 were obtained with the $0.5 \mathrm{~m}$ Centurian 18 at Wise Observatory (WC18, Brosch et al. 2008) and with the $0.9 \mathrm{~m}$ at West Mountain Observatory (WMO). WC18 uses a STL-6303E CCD with a pixel scale of $1{ }^{\prime \prime} 47$ and a $75^{\prime} \times 50^{\prime}$ field of view, and WMO uses a Finger Lakes PL-3041-UV CCD with a pixel scale of 0 ". 61 and a field of view of $21^{\prime} \times 21^{\prime}$. We also used data from the All-Sky Automated Survey for SuperNovae (ASAS-SN, Shappee et al. 2014). These data are from the first unit of ASAS-SN, Brutus, which consisted in 2012 of two $14 \mathrm{~cm}$ aperture Nikon telephoto lenses on a single mount in the
Faulkes Telescope North (Brown et al. 2013) enclosure on Mount Haleakala, Hawaii. ASAS-SN detectors are FLI ProLine CCD cameras, each with a Fairchild Imaging $2 \mathrm{k} \times 2 \mathrm{k}$ thinned $\mathrm{CCD}$, a $4.47 \times 4.47$ degree field of view, and a 7!! 8 pixel scale.

In addition, the 2014 campaign included imaging from CrAO, Fountainwood Observatory (FWO), and the Las Cumbres Observatory (LCO; Brown et al. 2013). The CrAO images were from the $0.7 \mathrm{~m}$ telescope equipped with an AP7p CCD with a pixel scale of 1 !'76 and a field of view of $15^{\prime} \times 15^{\prime}$. Observations from FWO were obtained with a $0.4 \mathrm{~m}$ telescope with an SBIG $8300 \mathrm{M}$ CCD. The field of view was $19^{\prime} \times 17^{\prime}$ and the pixel scale was 0 "' 35 . The LCO data were obtained using their worldwide network of $1 \mathrm{~m}$ telescopes in the Sloan ugriz bands.

\subsection{Data Processing and Light Curves}

The procedures we followed for reducing the data, producing calibrated light curves, and assessing uncertainties are described in detail by Fausnaugh et al. (2017). We provide a brief recapitulation here.

\subsubsection{Spectroscopy}

Two-dimensional spectra were reduced using standard IRAF tasks to deal with bias, flat field, sky subtraction, and wavelength calibration. An extraction window of $12^{\prime \prime}$ was used throughout. Cosmic ray removal was done using LA Cosmic (van Dokkum 2001). Flux calibration relied on observations of standard stars, usually Feige 34 and/or BD $+33^{\circ} 2642$ (Oke 1990).

We used the narrow [O III] $\lambda 5007$ emission line as an internal flux standard for both relative and absolute calibration. While narrow emission lines have been found to vary on long timescales (years to decades; e.g., Peterson et al. 2013), they are effectively constant in flux on BLR reverberation timescales (days to months). We identified all the individual spectra where the observer reported "clear" or "photometric" observing conditions. The $[\mathrm{O}$ III $] \lambda 5007$ flux was measured, and from 


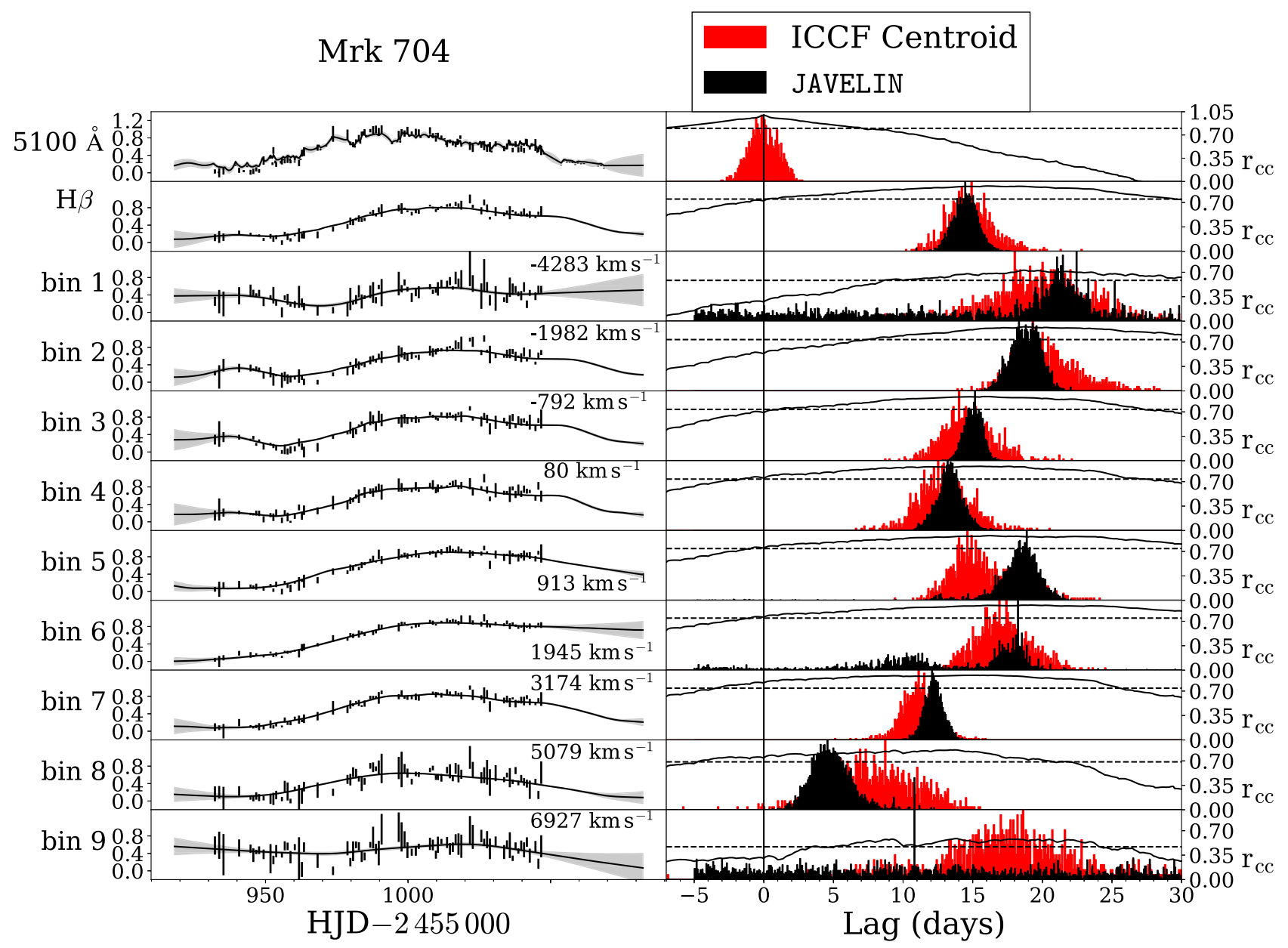

Figure 7. Left-hand panels: light curves for Mrk 704. The top panel shows the $5100 \AA$ A continuum light curve and the integrated $\mathrm{H} \beta$ light curve is shown underneath. Below are light curves in different Doppler velocity bins, starting with the far blueward wing and proceeding down the the far redward wing, with the flux-weighted average velocity of the bin labeled. Solid lines and shaded regions give the JAVELIN models and the $1 \sigma$ uncertainties. Right-hand panels: cross-correlations for Mrk 704. The solid line shows the cross-correlation function generated by cross-correlating the light curve to the immediate left with the $5100 \AA$ continuum light curve in the upper left panel; the upper right panel is the continuum autocorrelation function. The dashed lines are drawn at $0.8 r_{\max }$, where $r_{\max }$ is the peak of the crosscorrelation function, which occurs at $\tau_{\text {peak }}$; values above this threshold are used to compute the centroid $\tau_{\text {cent }}$. The cross-correlation centroid distribution (see Peterson et al. 1998) is shown in red and the JAVELIN posterior distribution of lags is shown in black.

these a mean and standard deviation was computed. Outliers greater than $3 \sigma$ from the mean were rejected and the mean and standard deviation were recomputed. The number of observations used for the calibration is given in Column (2) of Table 2 and the adopted [O III] $\lambda 5007$ fluxes appear in Column (3) of the same table. This provides the absolute flux calibration for the spectrophotometric observations. We note that the [O III] $\lambda 5007$ flux in NGC 5548 is in good agreement with the preliminary measurement we presented earlier (Peterson et al. 2013).

For each AGN, the spectra with the highest signal-to-noise ratios $(\mathrm{S} / \mathrm{Ns})$ and no obvious flaws are combined to form a reference spectrum, which is scaled to have the adopted [O III] $\lambda 5007$ flux. We then place all the individual spectra on the same relative flux by scaling each spectrum to the adopted [O III] $\lambda 5007$ flux. This is done using a Markov chain Monte Carlo code called mapspec (Fausnaugh 2017) which adjusts the flux, wavelength shift, and resolution of each individual spectrum to match that of the reference spectrum, as measured by the $[\mathrm{O} \mathrm{III]} \lambda 5007$ emission line profiles. This affords a factor of several improvement over the long-used method of van Groningen \& Wanders (1992), as assessed by the root-mean-square (rms) scatter of the [O III] $\lambda 5007$ flux across the full time series.

Once flux calibration is complete, we combine all $N$ spectra for each object to form a weighted mean spectrum

$$
\langle F(\lambda)\rangle=\frac{\sum_{i=1}^{N} F\left(\lambda, t_{i}\right) / \sigma^{2}\left(\lambda, t_{i}\right)}{\sum_{i=1}^{N} 1 / \sigma^{2}\left(\lambda, t_{i}\right)},
$$

where $F\left(\lambda, t_{i}\right)$ is the flux at epoch $t_{i}$ and $\sigma\left(\lambda, t_{i}\right)$ is the associated uncertainty. We also form an rms residual spectrum

$$
\sigma_{\mathrm{rms}}(\lambda)=\left\{\frac{1}{N-1} \sum_{i=1}^{N}\left[F\left(\lambda, t_{i}\right)-\langle F(\lambda)\rangle\right]^{2}\right\}^{1 / 2} .
$$

The mean and rms spectra for our sources are shown in Figures 1-6. The rms spectrum is of special value in this context, since the constant components (e.g., host-galaxy starlight, narrow emission lines) vanish, isolating the variable part of the spectrum. However, the total variability power contains contributions not only from intrinsic variability, but also from statistical fluctuations and/or measurement errors. We therefore attempt to isolate the intrinsic variability by 


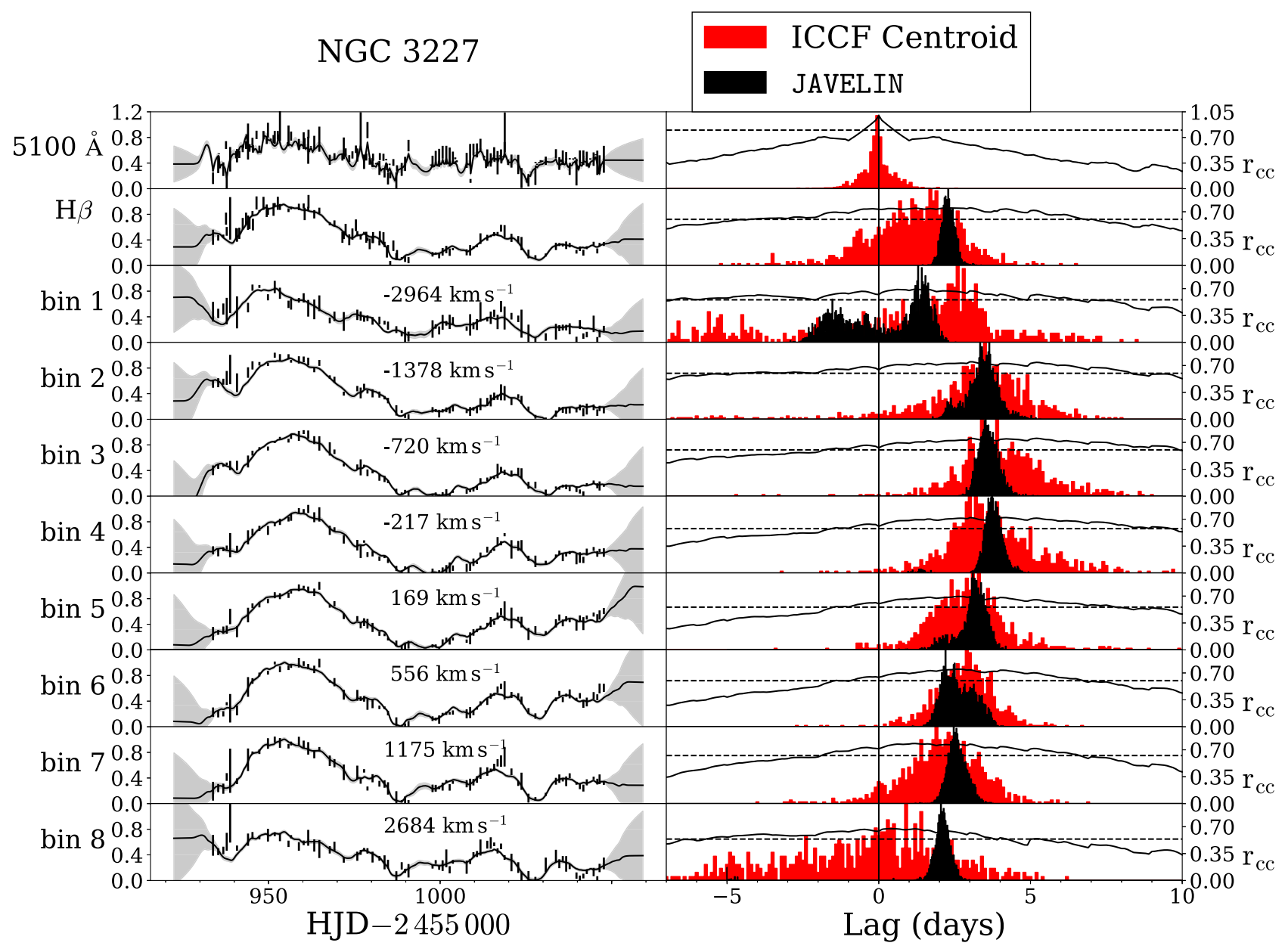

Figure 8. Light curves and cross-correlation functions for NGC 3227. The format is the same as in Figure 7.

minimizing the negative log-likelihood

$$
\begin{aligned}
-2 \ln \mathcal{L}= & \sum_{i=1}^{N} \frac{\left[F\left(\lambda, t_{i}\right)-\hat{F}(\lambda)\right]^{2}}{\sigma^{2}\left(\lambda, t_{i}\right)+\sigma_{\text {var }}^{2}(\lambda)} \\
& +\sum_{i=1}^{N} \ln \left[\sigma^{2}\left(\lambda, t_{1}\right)+\sigma_{\text {var }}^{2}(\lambda)\right],
\end{aligned}
$$

where $\hat{F}(\lambda)$ is the optimal average weighted by $\sigma^{2}\left(t_{i}\right)+\sigma_{\text {var }}^{2}$ and $\sigma_{\text {var }}(\lambda)$ is the intrinsic variability. We solve simultaneously for $\hat{F}(\lambda)$ and $\sigma_{\text {var }}(\lambda)$. Our estimator of the intrinsic variability $\sigma_{\text {var }}(\lambda)$ is also shown in Figures 1-6.

\subsubsection{Imaging}

Independent continuum light curves were generated for each bandpass for each set of imaging data using the image subtraction software package ISIS (Alard \& Lupton 1998; Alard 2000). We followed the procedures as described by Shappee \& Stanek (2011). First, we aligned the images using Sexterp (Siverd et al. 2012). We then created a reference image with ISIS for each AGN field by combining the images with the best seeing and lowest background counts; typically we used 5-15 images to construct the reference image. ISIS convolves the images of each AGN with a convolution kernel that is allowed to vary across the field in order to transform all the images to the same point-spread function (PSF) and background level. The reference image was convolved to match each individual frame and ISIS then subtracted each image from the convolved reference frame. The fluxes of the AGN and control stars to estimate errors were determined by fitting a PSF-weighted aperture over each source, thus producing a differential light curve.

\subsubsection{Construction of Light Curves}

A spectroscopic continuum light curve, nominally at $\sim 5100 \AA$ in the rest frame of each AGN, is formed by averaging the flux densities over the wavelength ranges given in Table 3 and shown as a shaded region in Figures 1-6. Our final continuum light curves are constructed by merging the differential $V$-band light curves with the $5100 \AA$ spectroscopic light curve by scaling multiplicatively (to match the variations) and shifting additively (to account for the different mean flux levels in each reference image) the differential continuum light curves. We found that the uncertainties on the differential light curves are systematically too small because ISIS takes into account only Poisson errors. To bypass this problem, we rescaled the errors based on measurements of other stars in the field of view, as described in detail by Fausnaugh et al. (2016).

The emission-line light curves are generated by interpolating a simple linear continuum underneath the emission lines using the windows given in Table 4 and integrating the flux above this continuum between the limits given in Table 3 and illustrated in Figures 1-6. These measurements are fairly crude, but are intended to capture the emission-line variations as 


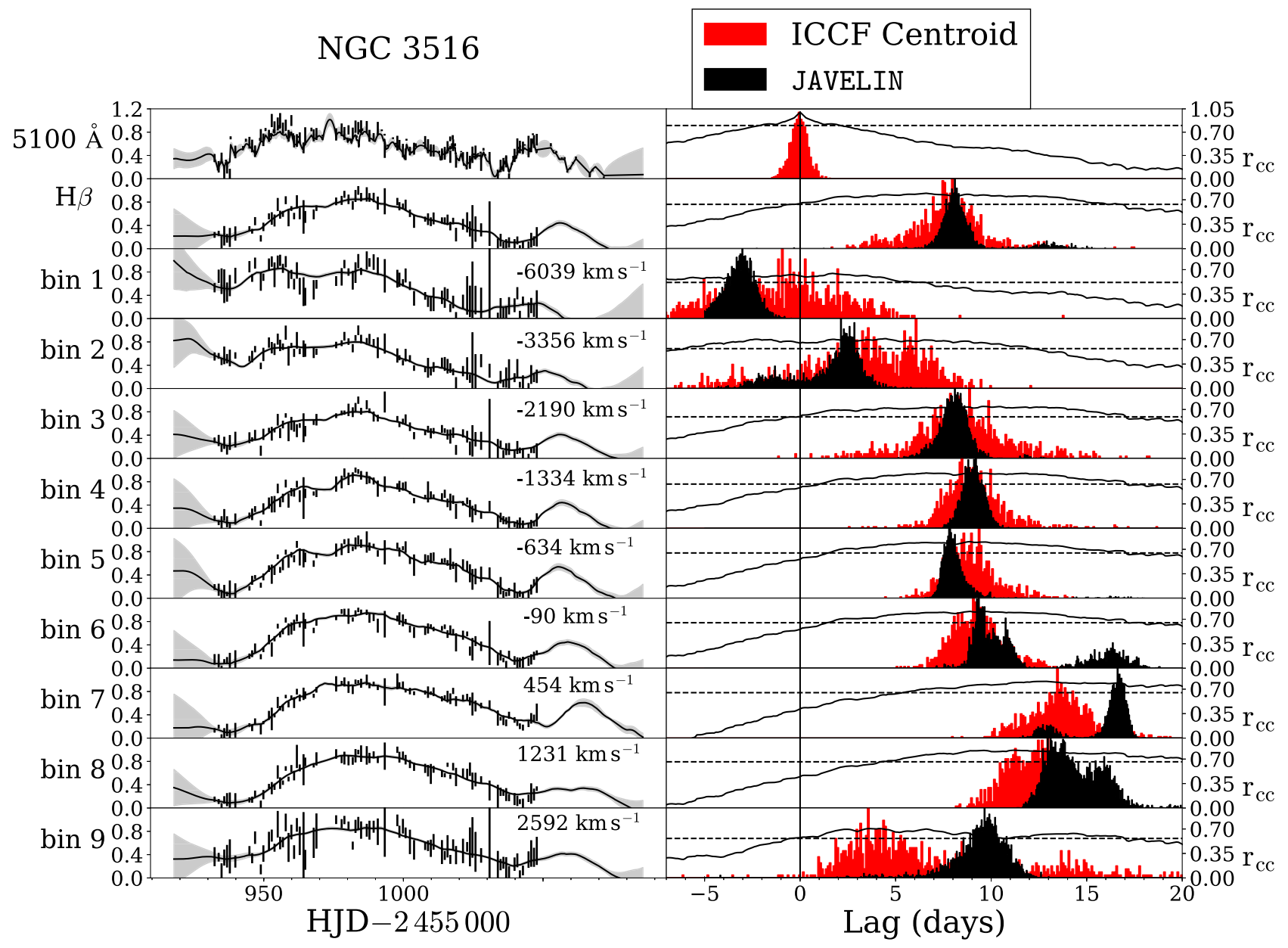

Figure 9. Light curves and cross-correlation functions for NGC 3516. The format is the same as in Figure 7.

opposed to all the emission-line flux. A more sophisticated treatment is deferred to a future paper. We estimate the uncertainties using a local linear interpolation method described in detail by Fausnaugh et al. (2017), which rescales the statistical uncertainties of the light curves so that they are consistent with the observed night-to-night scatter.

The final continuum and emission line light curves are given in Tables 5 and 6 respectively. All the light curves are shown in Figures 1-6. The statistical properties of the light curves are summarized in Table 7, including the number of observations $N_{\text {obs }}$, the uncertainty rescaling factor, median cadence $\Delta t_{\text {med }}$, mean flux $\langle F\rangle,\langle\mathrm{S} / \mathrm{N}\rangle$, excess variance $F_{\text {var }}=\sigma_{\text {var }} / \hat{F}$, where $\sigma_{\text {var }}$ and $\hat{F}$ are determined in the same way as in Equation (4) (after integrating over $\lambda$ to produce the light curves), and the significance

$$
(\mathrm{S} / \mathrm{N})_{\mathrm{var}}=\frac{\sigma_{\mathrm{var}}}{\bar{\sigma} \sqrt{2 / N_{\mathrm{obs}}}}
$$

at which variability is detected, where $\bar{\sigma}$ is the mean measurement uncertainty. Further details can be found in Fausnaugh et al. (2017).

\section{Time-series Analysis}

\subsection{Mean Emission-line Lags}

Our initial goal is to determine the mean timescale for the response of the $\mathrm{H} \beta$ emission line to continuum variations, which we later use to determine the mass of the central black hole.

The time series analysis is carried out using two common methodologies, interpolated cross-correlation (Gaskell \& Sparke 1986; Gaskell \& Peterson 1987; White \& Peterson 1994; Peterson et al. 1998, 2004) and the stochastic process modeling algorithm $\operatorname{JAVELIN}^{62}(\mathrm{Zu}$ et al. 2011). A more complete description of how we have employed these methods for such analysis is provided by Fausnaugh et al. (2017).

Results of the time-series analysis are given in Table 8 and shown graphically in the right-hand panels of Figures 7-11. It is interesting to notice that the three AGNs from Denney et al. (2009a), re-observed in this program, all have shorter lags than they did in 2007. In the case of NGC 3516, the factor-of-two decrease in the $\mathrm{H} \beta$ lag is consistent with the factor-of-four decrease in the AGN luminosity and the expected scaling relation $R_{\mathrm{BLR}} \propto L_{\mathrm{AGN}}^{1 / 2}$. In the case of NGC 3227, the $\mathrm{H} \beta$ lag also decreased by a factor of two from 2007, but the AGN luminosity is in fact slightly higher in 2012 and 2014. In 2012, NGC 5548 had been in a prolonged faint state for a few years (Peterson et al. 2013) and by 2013-14 heavy internal absorption became an important factor (Kaastra et al. 2014; De Rosa et al. 2015). In both 2012 and 2014 (Pei et al. 2017), the $\mathrm{H} \beta$ lag is found to be surprisingly short given the AGN luminosity at the time. The implications of this are not yet

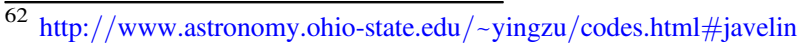




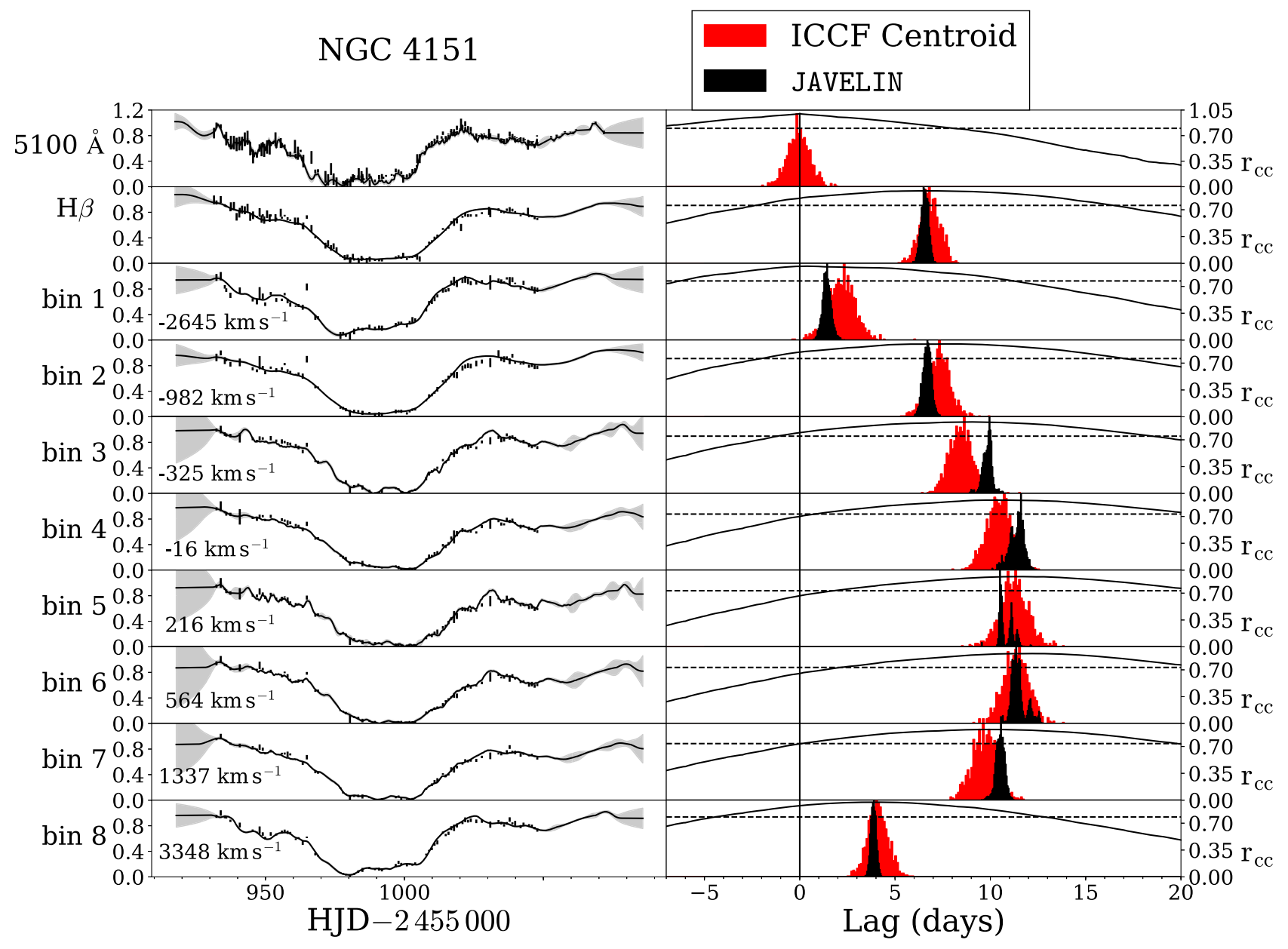

Figure 10. Light curves and cross-correlation functions for NGC 4151. The format is the same as in Figure 7.

clear, although it appears that increased absorption within the BLR plays some role. The 2014 data on NGC 3227 are quite marginal, and JAVELIN was unable to converge on a solution for the lag. The ICCF analysis, however, shows consistency with the 2012 results. In the case of NGC 4151, the $\mathrm{H} \beta$ lag is in good agreement with that obtained by Bentz et al. (2006a).

We note in passing that we also attempted to measure the variations of the He II $\lambda 4686$ line, which is clearly seen in the rms residual spectra of each source (Figures 1-6). Unfortunately this is a weak, low-contrast feature, and the measurements are very uncertain on account of the difficulties in defining the underlying continuum. Contamination of the spectra by the host-galaxy starlight is a significant problem in low-luminosity AGNs, and it needs to be modeled and subtracted for a reliable He II measurement. We defer this to a future paper.

\subsection{Velocity-resolved Lags}

The individual spectra are of high enough $\mathrm{S} / \mathrm{N}$ and sufficiently well-sampled in time that we can also divide each emission line into line-of-sight velocity bins to see if there are any indications of gross kinematic signature and, in the cases of NGC 3227, NGC 3516, and NGC 5548, compare these results with those obtained by Denney et al. (2009a). This is not a foolproof method of discerning the velocity field of the BLR as experience has shown that reverberation effects are quite subtle, and attempting to characterize an individual velocity bin with a single average lag could be misleading. While we must interpret the results cautiously, detection of a velocitydependent lag signature identifies good candidates for more ambitious attempts to determine the BLR structure and velocity field by either forward modeling (Pancoast et al. 2012, 2014; Grier et al. 2017) or regularization (Horne et al. 2004; Bentz et al. 2010a; Grier et al. 2013b; Skielboe et al. 2015). The results of measuring velocity-dependent lags are shown in the lower panels of Figures 7-11 in a format that can be easily compared with Figure 3 of Denney et al. (2009a) in Figures 12-16. We comment on each source individually:

Mrk 704. Figure 7 shows that the highest-velocity blueshifted and redshifted bins have large lag uncertainties, so we will disregard these. The remaining bins (Figure 12) show a local lag minimum around line center $(V=0)$. At higher redshifted velocities, the lags increase to a maximum at $\sim 2000 \mathrm{~km} \mathrm{~s}^{-1}$, then become smaller in the far wings. On the blueshifted side, the lags also increase, but we do not see a turnover toward smaller lags at higher velocity. A similar pattern with relatively small lags at the line center compared to the wings is seen in NGC 5548 in 2014 in $\mathrm{H} \beta$ (Pei et al. 2017) and also probably in Ly $\alpha$ (De Rosa et al. 2015). The BLR velocity field in Mrk 704 may well have multiple components, and requires more sophisticated modeling.

NGC 3227. As with Mrk 704, the highest-velocity bins have large errors and should be disregarded (Figure 8). The 


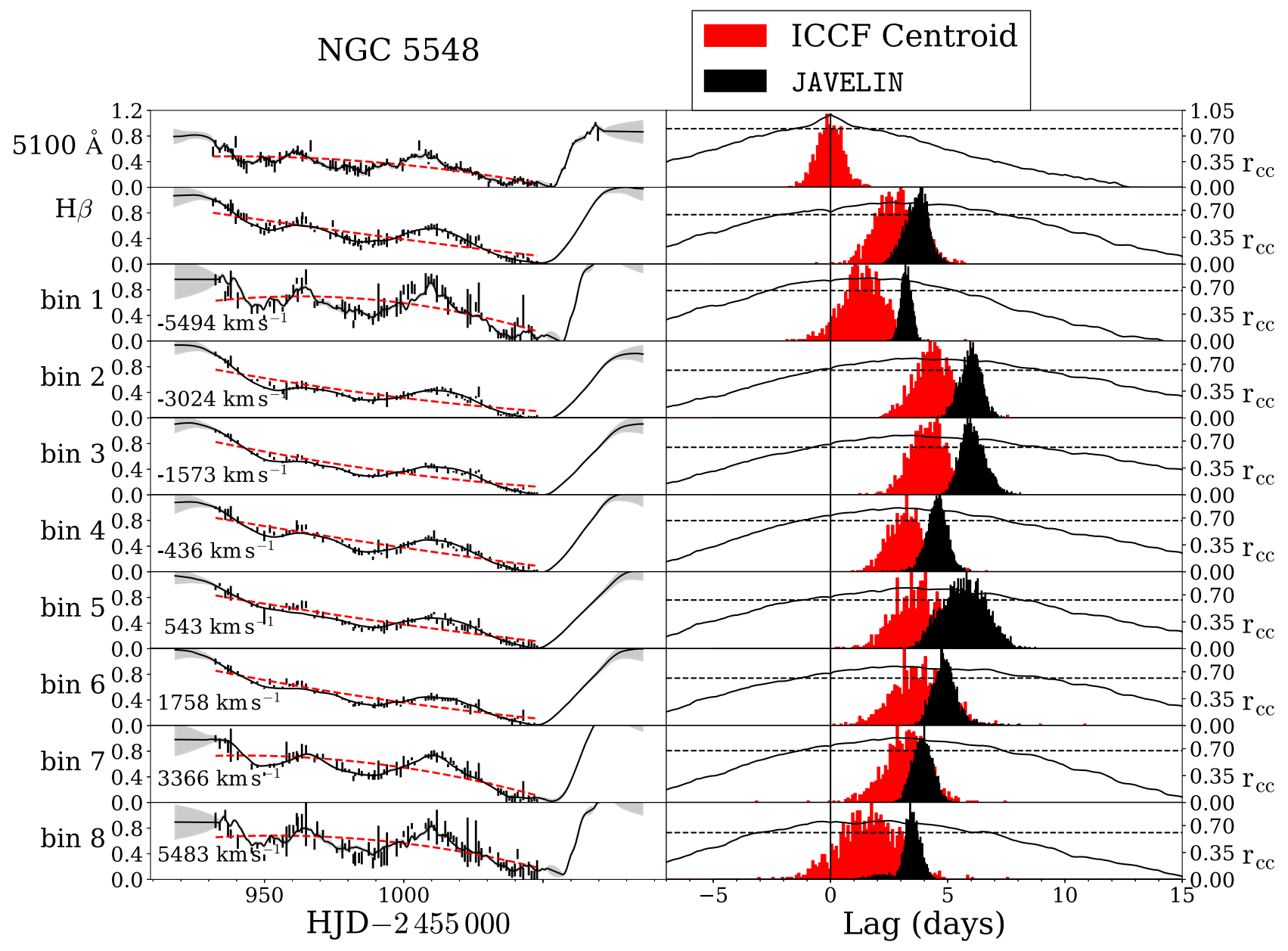

Figure 11. Light curves and cross-correlation functions for NGC 5548. The format is the same as in Figure 7. The dashed red lines show the results of second-order polynomial linear least-squares fits, which were used to detrend the light curves prior to calculating the interpolated cross-correlation function.

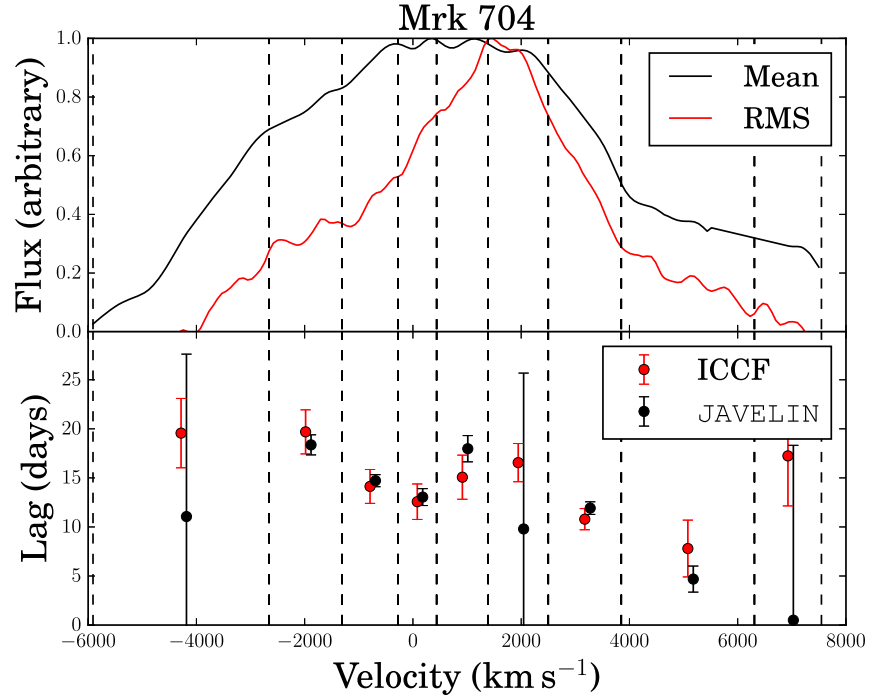

Figure 12. Top panel: mean (black) and rms residual (red) $\mathrm{H} \beta$ profiles for Mrk 704. The narrow-line component of $\mathrm{H} \beta$ and the [O III] $\lambda \lambda 4959$, 5007 lines have been modeled out of the mean spectrum. The vertical dashed lines show the velocity bins used to produce velocity-resolved light curves. The bin boundaries were chosen so that the total mean-spectrum flux in each bin is approximately the same. Bottom panel: lags measured for the emission in each velocity bin, with interpolated cross-correlation function lags shown in red and JAVELIN lags shown in black. The JAVELIN lags are offset by $+100 \mathrm{~km} \mathrm{~s}^{-1}$ for clarity.

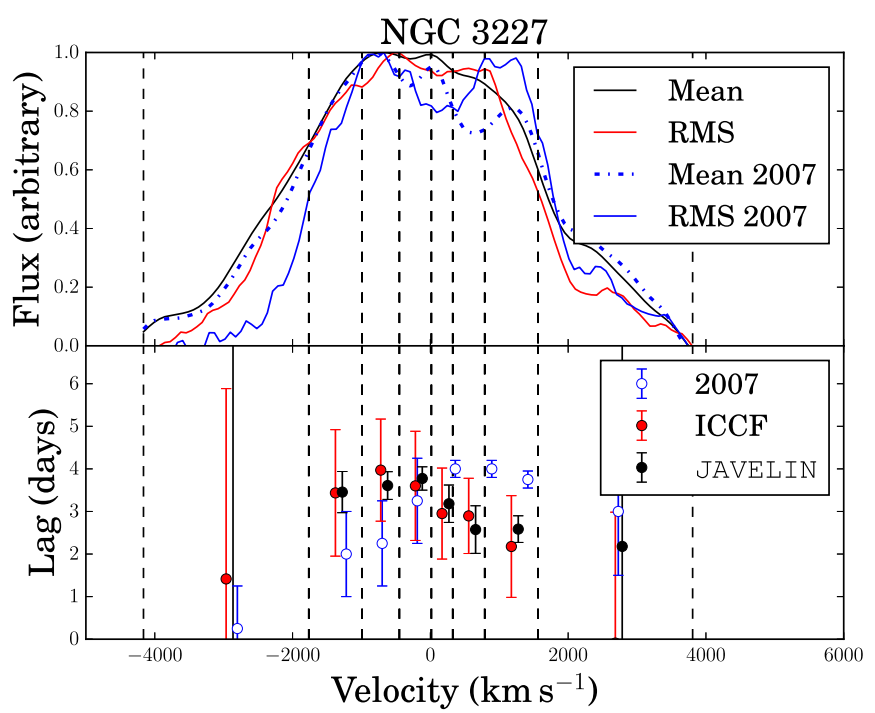

Figure 13. Upper panel: mean and rms residuals for $\mathrm{H} \beta$ in NGC 3227; lower panel: lag in each velocity bin. The format is the same as in Figure 12. In the upper panel, the 2007 mean (blue dashed line) and rms (blue solid line) $\mathrm{H} \beta$ profiles from Denney et al. (2009b) are shown. In the lower panel the lags from Denney et al. (2009a) are shown as open blue circles. Note that Denney et al. (2009a) used slightly different velocity bins than those defined here. 


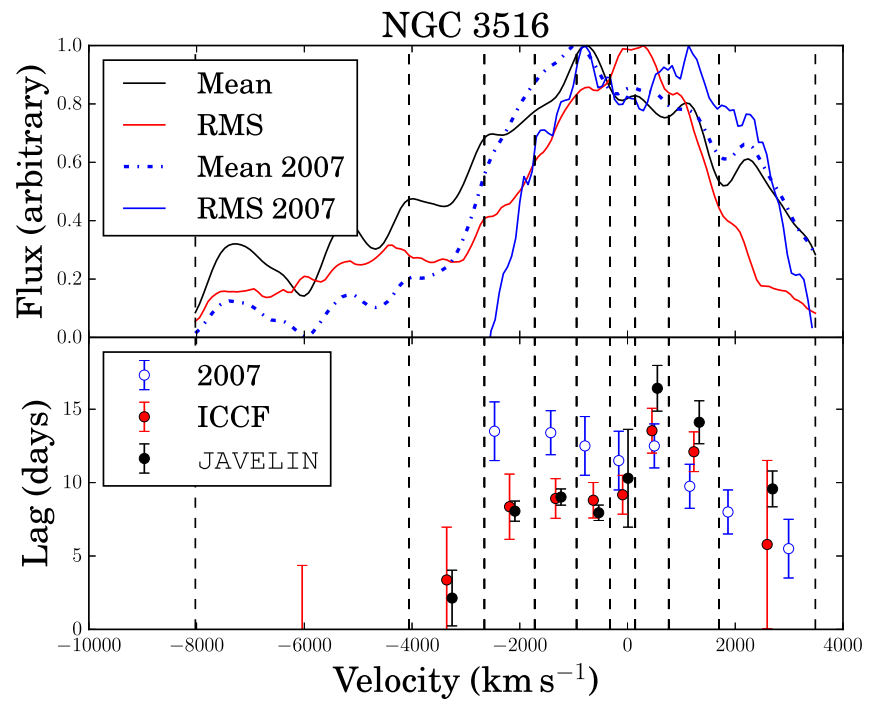

Figure 14. Upper panel: mean and rms residuals for $\mathrm{H} \beta$ in NGC 3516; lower panel: lag in each velocity bin. The format is the same as in Figure 12. In the upper panel, the 2007 mean (blue dashed line) and rms (blue solid line) $\mathrm{H} \beta$ profiles from Denney et al. (2009b) are shown. In the lower panel the lags from Denney et al. (2009a) are shown as open blue circles.

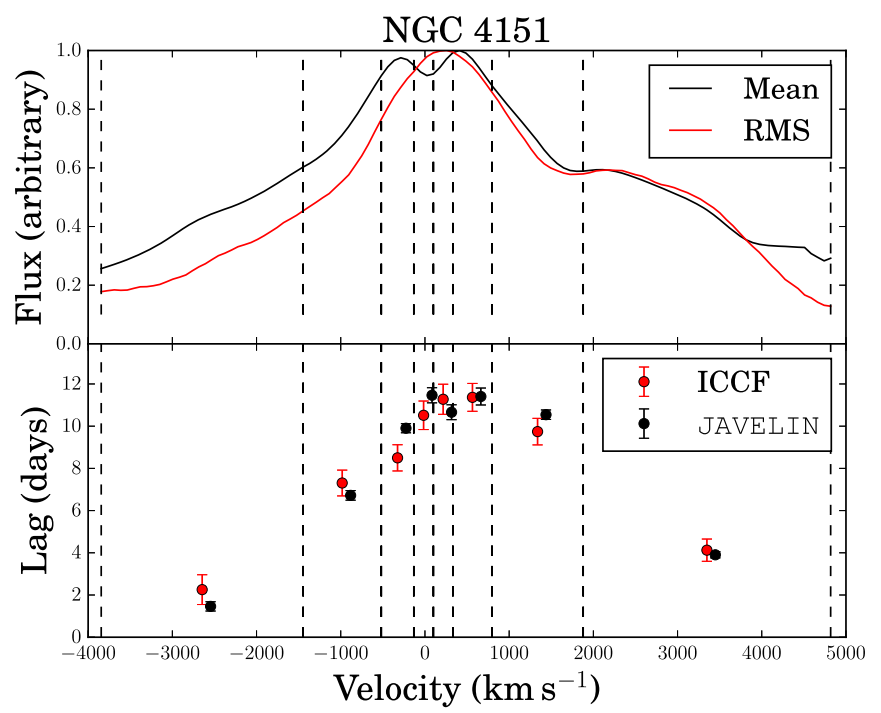

Figure 15. Upper panel: mean and rms residuals for $\mathrm{H} \beta$ in NGC 4151; lower panel: lag in each velocity bin. The format is the same as in Figure 12.

remaining bins show a pattern that suggests a virialized BLR (Figure 13), with large lags at the line center and shorter lags at higher positive and negative velocities. These can be compared with results from Denney et al. (2009a), which do not show a decrease in the lag at higher positive velocities. Lower lags at high negative velocity might be interpreted as evidence for outflow. There is no strong evidence for outflow in the 2012 data. Again, more sophisticated modeling will clarify the situation. As noted earlier, the 2014 data on this source are marginal and are not included in this analysis. We note that a very similar dependence of lag on velocity bin is seen from an independent RM campaign from 2017 (M.S. Brotherton 2018, private communication).

NGC 3516. In 2007 (Denney et al. 2009a), the highest positive velocities in the $\mathrm{H} \beta$ emission line showed the shortest lags, with the lags steadily increasing toward the line center and continuing to increase slightly to higher negative velocity

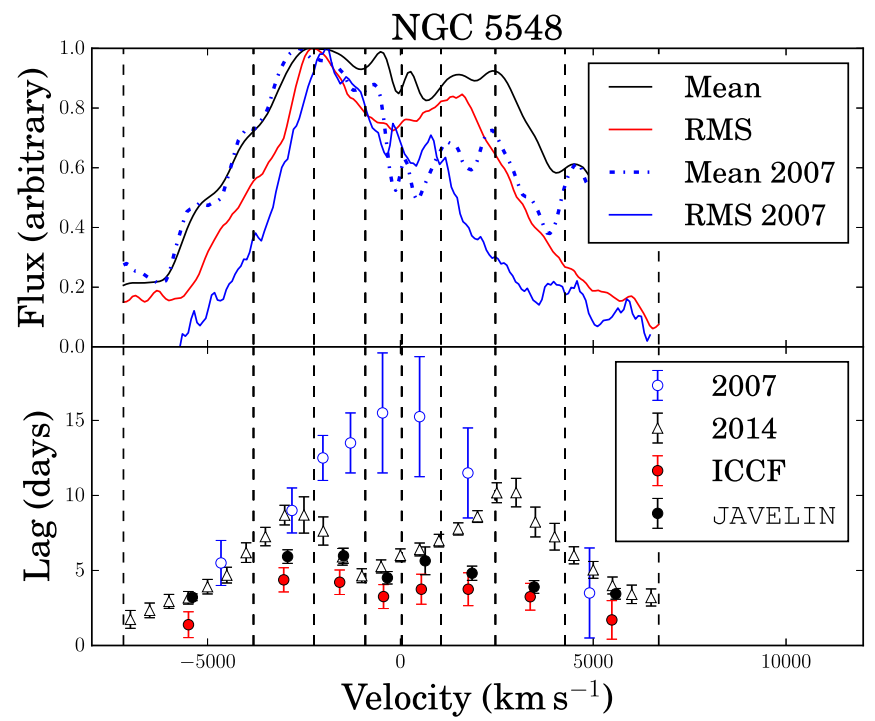

Figure 16. Upper panel: mean and rms residuals for $\mathrm{H} \beta$ in NGC 5548; lower panel: lag in each velocity bin. The format is the same as in Figure 12. In the upper panel, the 2007 mean (blue dashed line) and rms (blue solid line) $\mathrm{H} \beta$ profiles from Denney et al. (2009b) are shown. In the lower panel the lags from Denney et al. (2009a) are shown as open blue circles, while those from Pei et al. (2017) are shown as open black triangles.

(Figure 14). This behavior could be interpreted as an infall signature. In 2012, at least in the core of the line, this trend seems to be reversed.

$N G C$ 4151. On account of the brightness and favorable variability characteristics of NGC 4151 during this campaign, the results for this AGN are superb. The uncertainties in the lag for each velocity bin are quite small (Figure 10) and there is a very clear virial-like pattern where the largest lags are seen at the lowest velocities (Figure 15).

$N G C 5548$. Due to less favorable variability characteristics in 2012, the NGC 5548 results are not as clear as they were in either 2007 (Denney et al. 2009a) or in 2014 (Pei et al. 2017); the uncertainties in each velocity bin are comparatively large (Figure 11). The pattern as a function of wavelength seems to be quite similar to the complex pattern observed in 2014 (see Figure 10 of Pei et al. 2017) as well as in 2015 Lu et al. (2016). This is also similar to Mrk 704 (Figure 12), and possibly indicates a multicomponent BLR.

\section{Line Width and Black Hole Mass Calculation}

In order to compute the mass of the central black hole from Equation (1), we need to characterize the line width $\Delta V$ in addition to the mean emission-line lag $\tau$. The two line width measures commonly used for this are full width at half maximum (FWHM) and the line dispersion

$$
\sigma_{\text {line }}=\left[\frac{\int v^{2} P(v) d v}{\int P(v) d v}\right]^{1 / 2},
$$

which is the square root of the second moment of the line. The integral is over the line profile $P(v)$ as a function of line-ofsight (Doppler) velocity. There are practical advantages and disadvantages to each of these. The FWHM is usually trivial to measure, but presents problems when the data are noisy or the profiles are complex. The line dispersion, on the other hand, 
Table 9

Rest-frame $\mathrm{H} \beta$ Velocity Measurements

\begin{tabular}{|c|c|c|c|c|c|c|}
\hline \multirow[b]{2}{*}{ Object } & \multirow[b]{2}{*}{ Line } & \multicolumn{2}{|c|}{ rms Spectrum } & \multicolumn{2}{|c|}{ Mean Spectrum } & \multirow[b]{2}{*}{$\begin{array}{l}\text { Smoothing Width } \\
\left(\mathrm{km} \mathrm{s}^{-1}\right) \\
(7)\end{array}$} \\
\hline & & $\begin{array}{c}\sigma_{\text {line }} \\
\left(\mathrm{km} \mathrm{s}^{-1}\right) \\
(3)\end{array}$ & $\begin{array}{c}\text { FWHM } \\
\left(\mathrm{km} \mathrm{s}^{-1}\right) \\
(4)\end{array}$ & $\begin{array}{c}\sigma_{\text {line }} \\
\left(\mathrm{km} \mathrm{s}^{-1}\right) \\
(5)\end{array}$ & $\begin{array}{c}\text { FWHM } \\
\left(\mathrm{km} \mathrm{s}^{-1}\right) \\
(6)\end{array}$ & \\
\hline Mrk 704 & $\mathrm{H} \beta$ & $1860_{-130}^{+108}$ & $3406_{-240}^{+310}$ & $2650_{-3}^{+4}$ & $3502_{-30}^{+32}$ & 294 \\
\hline NGC 3227 (2012) & $\mathrm{H} \beta$ & $1368_{-37}^{+38}$ & $3837_{-107}^{+81}$ & $1402_{-2}^{+2}$ & $1602_{-17}^{+18}$ & 313 \\
\hline NGC 3227 (2014) & $\mathrm{H} \beta$ & $1428_{-106}^{+97}$ & $2236_{-387}^{+487}$ & $1301_{-3}^{+4}$ & $1324_{-17}^{+17}$ & 226 \\
\hline NGC 5548 & $\mathrm{H} \beta$ & $2772_{-34}^{+33}$ & $7038_{-110}^{+133}$ & $3056_{-4}^{+3}$ & $1094_{-9}^{+10}$ & 329 \\
\hline
\end{tabular}

Note. Columns 3 and 4 give the rms line width and FWHM in the rms spectrum. Columns 5 and 6 give the same but in the mean spectrum. All values are corrected for instrumental broadening and the smoothing introduced by the scaling algorithm (see Section 2.3.1); the FWHM of the Gaussian smoothing kernel is given in Column 7. Apart from Column 7, all values are reported in the rest frame.

Table 10

Black Hole Masses

\begin{tabular}{|c|c|c|c|c|c|}
\hline $\begin{array}{l}\text { Object } \\
\text { (1) }\end{array}$ & $\begin{array}{l}\tau_{\text {JAV }} \text { (days) } \\
\quad(2)\end{array}$ & $\begin{array}{c}\sigma_{\text {line }}\left(\mathrm{km} \mathrm{s}^{-1}\right) \\
(3)\end{array}$ & $\begin{array}{c}\log \mathrm{VP}\left(M_{\odot}\right) \text { (current) } \\
\text { (4) }\end{array}$ & $\begin{array}{c}\log \operatorname{VP}\left(M_{\odot}\right) \text { (previous) } \\
(5)\end{array}$ & $\begin{array}{c}\log M\left(M_{\odot}\right) \\
\text { (6) }\end{array}$ \\
\hline Mrk 704 & $14.19_{-0.79}^{+0.87}$ & $1860_{-130}^{+108}$ & $6.98 \pm 0.06$ & $\ldots$ & $7.63 \pm 0.14$ \\
\hline NGC 3227 (2012) & $2.30_{-0.20}^{+0.22}$ & $1368_{-37}^{+38}$ & $5.92 \pm 0.05$ & $6.21 \pm 0.04$ & $6.57 \pm 0.13$ \\
\hline NGC 3227 (2014) & $2.6 \pm 1.0$ & $1428_{-106}^{+97}$ & $6.01 \pm 0.19$ & $\ldots$ & $6.66 \pm 0.24$ \\
\hline NGC 3516 & $8.11_{-0.58}^{+0.75}$ & $2448_{-74}^{+63}$ & $6.99 \pm 0.05$ & $6.86 \pm 0.04$ & $7.63 \pm 0.13$ \\
\hline NGC 4151 & $6.59_{-0.21}^{+0.19}$ & $1940_{-22}^{+22}$ & $6.68 \pm 0.01$ & $6.93 \pm 0.04$ & $7.33 \pm 0.13$ \\
\hline NGC 5548 & $3.68_{-0.52}^{+0.43}$ & $2772_{-34}^{+33}$ & $6.74 \pm 0.06$ & $7.08 \pm 0.16$ & $7.39 \pm 0.14$ \\
\hline
\end{tabular}

Note. Columns 2 and 3 give the time delays measured by JAVELIN and line dispersion in the rms spectra. The log of the virial product (Equation (1)) is in Column 4, and previous determinations of the virial product are in Column 5; the previous NGC 3227 and NGC 3516 measurements are from Denney et al. (2010), NGC 4151 is from Bentz et al. (2006a), and the value for NGC 5548 is the mean and standard deviation of 16 reverberation results drawn from the literature. Column 6 gives the black hole mass based on the data from this campaign and assuming $\langle f\rangle=4.47 \pm 1.25$ (Woo et al. 2015).

requires attention to blending with other features, but is computationally well-defined for any profile. The two measures are not interchangeable, as their ratio varies with line shape, which is correlated with line width. There are compelling, but not conclusive, arguments that line dispersion is the better parameter for computing masses (Denney et al. 2013; Peterson 2014), so we use $\sigma_{\text {line }}$ in our mass calculations, but report both measures for both the mean and rms spectra in Table 9.

For the mass calculation, we use $\sigma_{\text {line }}$ from the rms spectra as the line width measure because the rms profile reflects the motions of the gas that is actually responding to the continuum flux variations. For the time delay, we use $\tau_{\text {JAV }}$, though the uncertainties in this quantity depend strongly on the assumption that all errors are Gaussian. In Table 10, we list the time lags and line widths adopted for each data set, and combine these to form the VP. To put the VPs on a calibrated mass scale, we adopt a mean scale factor of $f=4.47 \pm 1.25$ (Woo et al. 2015). The uncertainty in the scale factor is propagated into the masses given in Table 10 .

The virial product, $\mathrm{VP}=c \tau \Delta V^{2} / G$, is useful for comparing the masses derived in different reverberation programs because it involves only the two observables and physical constants. While the VPs obtained here are in reasonable agreement with earlier measurements (Columns 4 and 5 of Table 10), it is also clear that the formal uncertainties derived from the time delay and line width are too small. There is clearly some additional intrinsic scatter in the VP values beyond these formal estimates, indicating additional systematic uncertainties (perhaps due to the choice of integration windows or blended spectral components) and/or underestimated measure uncertainties. The previous value for NGC 5548 in the last row of Table 10 underscores this point: for this entry, we used the mean and standard deviation from 16 previous measurements of the VP based on $\mathrm{H} \beta$ reverberation, spanning the range $6.74<\log \mathrm{VP}<7.38$. The standard deviation of this distribution is $\Delta \log \mathrm{VP} \approx 0.15$, which is probably a good indicator of the true uncertainties in typical measurements. If this is true generally, then the VP values measured here are all in agreement with previous determinations.

\section{Conclusions}

We have presented new reverberation results for five bright local Seyfert galaxies. All five have been targets in previous reverberation campaigns. In two cases, Mrk 704 and NGC 4151, previous campaigns did not provide good measurements of the emission-line lags or black hole masses. Mrk 704 did not vary in a fashion conducive to reverberation (Barth et al. 2015), showing only monotonically decreasing light curves. Our new data on NGC 4151 are far more extensive than those from our 2005 campaign (Bentz et al. 2006a), which was abbreviated by poor weather. The other three AGNs-NGC 3227, NGC 3516, and NGC 5548have been targets in multiple previous reverberation campaigns, and were specifically included in this campaign to compare the velocity-dependent lags, which might be interpreted as indicators of the gross kinematics of the BLR, with 
previous results from our 2007 campaign (Denney et al. 2009a). In all three cases, the pattern of the lags as a function of velocity has changed. The most likely reason for this is that the BLR structure is probably complex and consists of multiple components-a disk and a wind, for example (e.g., StorchiBergmann et al. 2017) —and characterizing any particular velocity bin by a single lag is simply inadequate to describe the BLR structure and kinematics. The important point is that the apparent differences between the 2007 and 2012 results suggest that changes may occur over a BLR dynamical timescale. In a future paper, we will undertake a more detailed analysis of these data with the aim of determining the structure and kinematics of the BLR in these sources and determine whether or not the apparent changes are significant.

G.D.R., C.J.G., B.M.P., and R.W.P. are grateful for the support of the National Science Foundation through grant AST-1008882 to The Ohio State University. K.D.D., B.J.S., C.B.H., and J.L.V. acknowledge support by NSF Fellowships. M.C.B. gratefully acknowledges support from the NSF through CAREER grant AST-1253702. A.M.M. and D.M.S. acknowledge the support of NSF grants AST-1004756 and AST1009756. C.S.K. is supported by NSF grant AST-1515876. S.K. is supported at the Technion by the Kitzman Fellowship and by a grant from the Israel-Niedersachsen collaboration program. S.R. is supported at Technion by the Zeff Fellowship. S.G.S. acknowledges the support to $\mathrm{CrAO}$ in the frame of the "CosmoMicroPhysics" Target Scientific Research Complex Programme of the National Academy of Sciences of Ukraine (2007-2012). M.V. gratefully acknowledges support from the Danish Council for Independent Research via grant no. DFF 4002-00275. V.T.D. acknowledges the support of the Russian Foundation of Research (RF project no. 12-02-01237-a). The CrAO CCD cameras were purchased through the US Civilian Research and Development for Independent States of the Former Soviet Union (CRDF) awards UP1-2116 and UP12549-CR-03. This research has been partly supported by the Grants-in-Aid of Scientific Research (17104002, 20041003, 21018003, 21018005, 22253002, and 22540247) of the Ministry of Education, Science, Culture and Sports of Japan. This research has made use of the NASA/IPAC Extragalactic Database (NED), which is operated by the Jet Propulsion Laboratory, California Institute of Technology, under contract with the National Aeronautics and Space Administration.

Software: IRAF (Tody 1986, 1993), LA Cosmic (van Dokkum 2001), mapspec (Fausnaugh 2017), ISIS (Alard \& Lupton 1998; Alard 2000), Sexterp (Siverd et al. 2012), JAVELIN (Zu et al. 2011).

\section{ORCID iDs}

G. De Rosa (1) https://orcid.org/0000-0003-3242-7052

C. J. Grier (iD https://orcid.org/0000-0001-9920-6057

B. M. Peterson (iD https://orcid.org/0000-0001-6481-5397

Keith Horne (iD https://orcid.org/0000-0003-1728-0304

M. C. Bentz (D) https://orcid.org/0000-0002-2816-5398

C. S. Kochanek (iD https://orcid.org/0000-0001-6017-2961

R. W. Pogge (iD https://orcid.org/0000-0003-1435-3053

M. Vestergaard (iD https://orcid.org/0000-0001-9191-9837

J. Bae (ib https://orcid.org/0000-0001-7258-770X

A. J. Barth (1) https://orcid.org/0000-0002-3026-0562

T. G. Beatty (iD https://orcid.org/0000-0002-9539-4203

T. A. Boroson (ib https://orcid.org/0000-0001-9481-1805
J. S. Brown (iD https://orcid.org/0000-0002-1885-6419

M. S. Brotherton (iD https://orcid.org/0000-0002-1207-0909

C. T. Coker (i) https://orcid.org/0000-0002-9954-7887

K. V. Croxall (D) https://orcid.org/0000-0002-5258-7224

M. Eracleous (iD https://orcid.org/0000-0002-3719-940X

K. Hawkins (ib https://orcid.org/0000-0002-1423-2174

C. B. Henderson (iD https://orcid.org/0000-0001-8877-9060

T. W.-S. Holoien (iD https://orcid.org/0000-0001-9206-3460

C. D. Laney (i) https://orcid.org/0000-0003-2967-110X

J. W. Moody (iD https://orcid.org/0000-0002-8874-0534

C. A. Onken (iD https://orcid.org/0000-0003-0017-349X

A. Pancoast (i) https://orcid.org/0000-0003-1065-5046

M. Penny (i) https://orcid.org/0000-0001-7506-5640

R. Poleski (i) https://orcid.org/0000-0002-9245-6368

J.-L. Prieto (i) https://orcid.org/0000-0003-0943-0026

A. M. Price-Whelan (i) https://orcid.org/0000-00030872-7098

R. M. Roettenbacher (iD https://orcid.org/0000-00029288-3482

D. M. Skowron (1D https://orcid.org/0000-0002-2335-1730

G. Somers (iD https://orcid.org/0000-0002-9322-0314

D. J. Stevens (iD https://orcid.org/0000-0002-5951-8328

J. Tayar (i) https://orcid.org/0000-0002-4818-7885

S. Valenti (iD https://orcid.org/0000-0001-8818-0795

C. Villforth (ib https://orcid.org/0000-0002-8956-6654

H. Winkler (ib https://orcid.org/0000-0003-2662-0526

\section{References}

Alard, C. 2000, A\&AS, 144, 363

Alard, C., \& Lupton, R. H. 1998, ApJ, 503, 325

Andrillat, Y. 1968, AJ, 73, 862

Antonucci, R. R. J., \& Cohen, R. D. 1983, ApJ, 271, 564

Bahcall, J. N., Kozlovsky, B.-Z., \& Salpeter, E. E. 1972, ApJ, 171, 467

Barth, A. J., Bennert, V. N., Canalizo, G., et al. 2015, ApJS, 217, 26

Barth, A. J., Nguyen, M. L., Malkan, M. A., et al. 2011a, ApJ, 732, 121

Barth, A. J., Pancoast, A., Bennert, V. N., et al. 2013, ApJ, 769, 128

Barth, A. J., Pancoast, A., Thorman, S. J., et al. 2011b, ApJL, 743, L4

Batiste, M., Bentz, M., Raimundo, S. I., et al. 2017, ApJ, 838, 10

Bentz, M. C., Batiste, M., Seals, J., et al. 2016a, ApJ, 831, 2

Bentz, M. C., Cackett, E. M., Crenshaw, D. M., et al. 2016b, ApJ, 830, 136

Bentz, M. C., Denney, K. D., Cackett, E. M., et al. 2006a, ApJ, 651, 775

Bentz, M. C., Denney, K. D., Cackett, E. M., et al. 2007, ApJ, 662, 205

Bentz, M. C., Denney, K. D., Grier, C. J., et al. 2013, ApJ, 767, 149

Bentz, M. C., Horenstein, D., Bazhaw, C., et al. 2014, ApJ, 796, 8

Bentz, M. C., Horne, K., Barth, A. J., et al. 2010a, ApJL, 720, L46

Bentz, M. C., \& Katz, S. 2015, PASP, 127, 67

Bentz, M. C., Peterson, B. M., Netzer, H., Pogge, R. W., \& Vestergaard, M. 2009a, ApJ, 697, 160

Bentz, M. C., Peterson, B. M., Pogge, R. W., Vestergaard, M., \& Onken, C. A. 2006b, ApJ, 644, 133

Bentz, M. C., Walsh, J. L., Barth, A. J., et al. 2009b, ApJ, 705, 199

Bentz, M. C., Walsh, J. L., Barth, A. J., et al. 2010b, ApJ, 716, 993

Blandford, R. D., \& McKee, C. F. 1982, ApJ, 255, 419

Boksenberg, A., \& Netzer, H. 1977, ApJ, 212, 37

Brosch, N., Polishook, D., Shporer, A., et al. 2008, Ap\&SS, 314, 163 Brown, T. M., Baliber, N., Bianco, F. B., et al. 2013, PASP, 125, 1031

Collin-Souffrin, S. 1980, in Variability of Stars and Galaxies: Proc. Fifth European Regional Meeting in Astronomy, ed. P. Ledoux (Liège: Institut d'Astrophysique), C.1.11

Dasyra, K. M., Tacconi, L. J., Davies, R. I., et al. 2007, ApJ, 657, 102

Davies, R. I., Thomas, J., Genzel, R., et al. 2006, ApJ, 646, 754

Denney, K. D., Bentz, M. C., Peterson, B. M., et al. 2006, ApJ, 653, 152

Denney, K. D., Peterson, B. M., Pogge, R. W., et al. 2009a, ApJL, 704, L80

Denney, K. D., Peterson, B. M., Pogge, R. W., et al. 2010, ApJ, 721, 715

Denney, K. D., Pogge, R. W., Assef, R. J., et al. 2013, ApJ, 775, 60

Denney, K. D., Watson, L. C., Peterson, B. M., et al. 2009b, ApJ, 702, 1353

De Rosa, G., Peterson, B. M., Ely, J., et al. 2015, ApJ, 806, 128

Du, P., Hu, C., Lu, K.-X., et al. 2014, ApJ, 782, 45

Du, P., Hu, C., Lu, K.-X., et al. 2015, ApJ, 806, 22 
Du, P., Lu, K.-X., Hu, C., et al. 2016, ApJ, 820, 27

Edelson, R., Gelbord, J. M., Horne, K., et al. 2015, ApJ, 806, 129 Fausnaugh, M. M. 2017, PASP, 129, 024007

Fausnaugh, M. M., Denney, K. D., Barth, A. J., et al. 2016, ApJ, 821, 56 Fausnaugh, M. M., Grier, C. J., Bentz, M. C., et al. 2017, ApJ, 840, 97 Ferrarese, L., \& Merritt, D. M. 2000, ApJL, 539, L9

Ferrarese, L., Pogge, R. W., Peterson, B. M., et al. 2001, ApJL, 555, L79 Foltz, C. B., Peterson, B. M., Capriotti, E. R., et al. 1981, ApJ, 250, 508 Gaskell, C. M., \& Peterson, B. M. 1987, ApJS, 65, 1

Gaskell, C. M., \& Sparke, L. S. 1986, ApJ, 305, 175

Gebhardt, K., Bender, R., Bower, G., et al. 2000a, ApJL, 539, L13

Gebhardt, K., Bender, R., Bower, G., et al. 2000b, ApJL, 543, L5

Goad, M. R., Korista, K. T., De Rosa, G., et al. 2016, ApJ, 824, 11

Graham, A. W., Onken, C. A., Athanassoula, E., \& Combes, F. 2011, MNRAS, 412, 2211

Grier, C. J., Martini, P., Watson, L. C., et al. 2013a, ApJ, 773, 90

Grier, C. J., Pancoast, A., Barth, A., et al. 2017, ApJ, 849, 146

Grier, C. J., Peterson, B. M., Horne, K., et al. 2013b, ApJ, 764, 47

Grier, C. J., Peterson, B. M., Pogge, R. W., et al. 2012a, ApJL, 744, L4

Grier, C. J., Peterson, B. M., Pogge, R. W., et al. 2012b, ApJ, 755, 60

Gültekin, K., Richstone, D. O., Gebhardt, K., et al. 2009, ApJ, 698, 198

Hicks, E. K. S., \& Malkan, M. A. 2008, ApJS, 174, 31

Horne, K., Peterson, B. M., Collier, S. J., \& Netzer, H. 2004, PASP, 116, 465

Kaastra, J. S., Kriss, G. A., Cappi, M., et al. 2014, Sci, 345, 64

Kaspi, S., Maoz, D., Netzer, H., et al. 2005, ApJ, 629, 61

Kaspi, S., Smith, P. S., Netzer, H., et al. 2000, ApJ, 533, 631

King, A. L., Martini, P., Davis, T. M., et al. 2015, MNRAS, 453, 1701

Kollatschny, W. 2003, A\&A, 407, 461

Kollatschny, W., Fricke, K. J., Schleicher, H., \& Yorke, H. W. 1981, A\&A, 102, L23

Laor, A. 1998, ApJL, 505, L83

Lu, K.-X., Du, P., Hu, C., et al. 2016, ApJ, 827, 118

Magorrian, J., Tremaine, S., Richstone, D., et al. 1998, AJ, 115, 2285

Mathur, S., Gupta, A., Page, K., et al. 2017, ApJ, 846, 55

McConnell, N. J., \& Ma, C.-P. 2013, ApJ, 764, 184

McConnell, N. J., Ma, C.-P., Gebhardt, K., et al. 2011, Natur, 469, 215

Nelson, C. H., Green, R. F., Bower, G., Gebhardt, K., \& Weistrop, D. 2004, ApJ, 615, 645

Oke, J. B. 1990, AJ, 99, 1621

Onken, C. A., Ferrarese, L., Merritt, D., et al. 2004, ApJ, 615, 645

Onken, C. A., Valluri, M., Brown, J. S., et al. 2014, ApJ, 791, 37

Pancoast, A., Brewer, B. J., Treu, T., et al. 2012, ApJ, 754, 49

Pancoast, A., Brewer, B. J., Treu, T., et al. 2014, MNRAS, 445, 3073

Park, D., Kelly, B. C., Woo, J.-H., \& Treu, T. 2012, ApJS, 203, 6

Pastoriza, M., \& Gerola, H. 1970, ApL, 6, 155

Pei, L., Barth, A. J., Aldering, G. S., et al. 2014, ApJ, 795, 38
Pei, L., Fausnaugh, M. M., Barth, A. J., et al. 2017, ApJ, 837, 131

Peterson, B. M. 1988, PASP, 100, 18

Peterson, B. M. 1993, PASP, 105, 247

Peterson, B. M. 2011, arXiv:1109.4181

Peterson, B. M. 2014, SSRv, 183, 253

Peterson, B. M., Denney, K. D., De Rosa, G., et al. 2013, ApJ, 779, 109

Peterson, B. M., Ferrarese, L., Gilbert, K. M., et al. 2004, ApJ, 613, 682

Peterson, B. M., Foltz, C. B., Byard, P. L., \& Wagner, R. M. 1982, ApJS, 49, 469

Peterson, B. M., \& Wandel, A. 1999, ApJL, 521, L95

Peterson, B. M., \& Wandel, A. 2000, ApJL, 540, L13

Peterson, B. M., Wanders, I., Horne, K., et al. 1998, PASP, 110, 660

Pronik, I. I. 1980, in Variability of Stars and Galaxies: Proc. Fifth European Regional Meeting in Astronomy, ed. P. Ledoux (Liège': Institut d'Astrophysique), C.1.1

Schlafly, E. F., \& Finkbeiner, D. P. 2011, ApJ, 737, 103

Schulz, H., \& Rafanelli, R. 1981, A\&A, 103, 216

Shappee, B. J., Prieto, J.-L., Grupe, D., et al. 2014, ApJ, 788, 48

Shappee, B. J., \& Stanek, K. Z. 2011, ApJ, 733, 124

Shen, Y., Brandt, W. N., \& Dawson, K. S. 2015, ApJS, 216, 44

Siverd, R. J., Beatty, T. G., Pepper, J., et al. 2012, ApJ, 761, 123

Skielboe, A., Pancoast, A., Treu, T., et al. 2015, MNRAS, 454, 144

Starkey, D., Horne, K., Fausnaugh, M. M., et al. 2017, ApJ, 835, 65

Storchi-Bergmann, T., Schimoia, J. S., Peterson, B. M., et al. 2017, ApJ, 835,236

Tody, D. 1986, Proc. SPIE, 627, 733

Tody, D. 1993, in ASP Conf. Ser. 52, Astronomical Data Analysis Software and Systems II, ed. R. J. Hanisch, R. J. V. Brissenden, \& J. Barnes (San Francisco, CA: ASP), 173

Tohline, J. E., \& Osterbrock, D. E. 1976, ApJL, 210, L117

Tonry, J. L., Dressler, A., Blakeslee, J. P., et al. 2001, ApJ, 546, 681

Tremaine, S., Gebhardt, K., Bender, R., et al. 2002, ApJ, 574, 740

Ulrich, M. H., Boksenberg, A., Bromage, G. E., et al. 1984, MNRAS, 206, 221

van Dokkum, P. G. 2001, PASP, 113, 1420

van Groningen, E., \& Wanders, I. 1992, PASP, 104, 700

Vestergaard, M., Denney, K., Fan, X., et al. 2011, in Narrow-Line Seyfert 1 Galaxies and Their Place in the Universe, ed. L. Foschini et al. (Trieste: POS), 38

Wandel, A., Peterson, B. M., \& Malkan, M. A. 1999, ApJ, 526, 579

Wang, J.-M., Du, P., Hu, C., et al. 2014, ApJ, 793, 108

Waters, T., Kashi, A., Proga, D., et al. 2016, ApJ, 827, 53

White, R. J., \& Peterson, B. M. 1994, PASP, 106, 879

Woo, J.-H., Treu, T., Barth, A. J., et al. 2010, ApJ, 716, 269

Woo, J.-H., Yoon, Y., Park, S., \& Kim, S. C. 2015, ApJ, 801, 38

Zu, Y., Kochanek, C. S., \& Peterson, B. M. 2011, ApJ, 735, 80 